\title{
Association between funding source, methodological quality and research outcomes in randomized controlled trials of synbiotics, probiotics and prebiotics added to infant formula: A Systematic Review
}

Mary N Mugambi ${ }^{1 *}$, Alfred Musekiwa ${ }^{2}$, Martani Lombard ${ }^{1}$, Taryn Young ${ }^{2}$ and Reneé Blaauw ${ }^{1}$

\begin{abstract}
Background: There is little or no information available on the impact of funding by the food industry on trial outcomes and methodological quality of synbiotics, probiotics and prebiotics research in infants. The objective of this study was to compare the methodological quality, outcomes of food industry sponsored trials versus non industry sponsored trials, with regards to supplementation of synbiotics, probiotics and prebiotics in infant formula.

Methods: A comprehensive search was conducted to identify published and unpublished randomized clinical trials (RCTs). Cochrane methodology was used to assess the risk of bias of included RCTs in the following domains: 1) sequence generation; 2) allocation concealment; 3) blinding; 4) incomplete outcome data; 5) selective outcome reporting; and 6) other bias. Clinical outcomes and authors' conclusions were reported in frequencies and percentages. The association between source of funding, risk of bias, clinical outcomes and conclusions were assessed using Pearson's Chi-square test and the Fisher's exact test. A p-value $<0.05$ was statistically significant.

Results: Sixty seven completed and 3 on-going RCTs were included. Forty (59.7\%) were funded by food industry, 11 (16.4\%) by non-industry entities and 16 (23.9\%) did not specify source of funding. Several risk of bias domains, especially sequence generation, allocation concealment and blinding, were not adequately reported. There was no significant association between the source of funding and sequence generation, allocation concealment, blinding and selective reporting, majority of reported clinical outcomes or authors' conclusions. On the other hand, source of funding was significantly associated with the domains of incomplete outcome data, free of other bias domains as well as reported antibiotic use and conclusions on weight gain.

Conclusion: In RCTs on infants fed infant formula containing probiotics, prebiotics or synbiotics, the source of funding did not influence the majority of outcomes in favour of the sponsors' products. More non-industry funded research is needed to further assess the impact of funding on methodological quality, reported clinical outcomes and authors' conclusions.
\end{abstract}

Keywords: Synbiotics, Probiotics, Prebiotics, Funding source, Methodological quality

\footnotetext{
* Correspondence: nkmugambi@hotmail.com

'Division of Human Nutrition, Faculty of Medicine and Health Sciences, Stellenbosch University, P.O Box 19063, Tygerberg 7505, South Africa Full list of author information is available at the end of the article
} 


\section{Background}

There are numerous studies that explore the relationship between industrial sponsorship of biomedical research and published outcomes [1]. Several reviews have documented how trials funded by industry are more likely to report results in favour of the sponsor's products [2-5]. These reviews focused on trials sponsored by the pharmaceutical industry. Few reviews have explored the impact of funding by the food industry on outcomes of research trials [6,7]. A review by Nkansah et al. also found that majority of trials investigating the effects of calcium supplementation in healthy children were industry funded and all supported calcium supplementation, in favour of the sponsor [8]. Similarly, a review by Lesser et al. found that scientific nutrition related articles (intervention trials, observational studies and scientific reviews) on common consumed beverages (soft drinks, juice, milk) funded by the food industry, were more likely to be favourable to the sponsor than articles that did not have industry funding [6].

Reporting only positive outcomes in a research trial significantly reduces a sponsors' financial risk. Pressure to show a food product causes favourable outcomes in a specific population, may result in biases in trial design (methodology) and reporting of outcomes in industry sponsored research. This type of bias in nutrition research could adversely affect public health. Results from nutrition research also influence policy formulation, professional dietary guidelines, design of public health interventions and regulation of food product health claims. In addition, findings from nutrition research often receive publicity from the media, which influences consumer behaviour [6].

More studies are needed to explore the relationship between the food industry and nutrition research [7]. There is little or no information available on the impact of funding by the food industry on trial outcomes and methodological quality of synbiotics, probiotics and prebiotics research in infants. There are no systematic reviews that have explored if sources of funding affects outcomes and methodological quality of randomized controlled trials (RCTs) conducted on infants given probiotics, prebiotics or synbiotics supplemented infant formula.

Probiotics are defined as "live microorganisms" which when administered in adequate amounts may confer a health benefit to the host [9]. The main probiotic organisms that are currently used worldwide belong to the genera Lactobacillus and Bifidobacteria and are found in the gastrointestinal microflora [9]. The probiotics preparations of interest for this review are those added to infant formulas. Prebiotics are non- digestible food ingredients that may benefit the host by selectively stimulating the growth and/or activity of one or a limited number of bacteria in the colon and improving the host's health [10-12]. The most widely studied prebiotics are galactooligosaccharides (GOS), inulin and fructooligosaccharide (FOS) [13,14]. GOS, FOS and inulin are added to different foods as fat and sugar replacements to improve texture or for their functional benefits $[10,15,16]$. When probiotics and prebiotics are administered simultaneously, the combination is termed Synbiotics.

The aim of this review was to explore whether financial sponsorship by the food industry affects outcomes and methodological quality of trials on synbiotics, probiotics or prebiotics used in infants. Methodological quality may be compromised when insufficient information is provided regarding sequence generation, allocation concealment, blinding, bias introduced from other sources and incomplete outcome reporting.

\section{Objective}

The objective of this systematic review was to compare the methodological quality and outcomes of food industry sponsored trials versus non industry sponsored trials with regards to supplementation of synbiotics, probiotics and prebiotics in infant formula.

\section{Hypothesis}

The source of funding in research trials using probiotics, prebiotics or synbiotics supplemented formula in infants is associated with outcomes in favour of the sponsor's products and authors' conclusions.

\section{Methods}

\section{Criteria for considering studies for this review Types of studies}

All randomized controlled trials (RCTs) conducted from 1980 to 2012 (irrespective of language) on synbiotics, probiotics, or prebiotics added to infant formula were included. Study participants were healthy full term infants (>37 weeks gestation or $>2.5 \mathrm{~kg}$ birth weight, 0-12 months old), preterm infants (born $<37$ weeks gestation), low birth weight $(<2.5 \mathrm{~kg}$ at birth) and extreme low birth weight infants $(<1000 \mathrm{~g}$ at birth). Infants were fed either infant formula (preterm or full term formula), mixed feeds (breast milk with infant formula) with added synbiotics, probiotics or prebiotics or conventional infant formula with or without placebo. RCTs were excluded if they included infants with cardiac defects, pulmonary diseases, gastrointestinal diseases, major congenital abnormalities or chromosomal abnormalities. Commentaries, editorials, letters to the editor and studies that were not RCTs were excluded.

\section{Types of outcome}

The outcomes included: 1) Source of funding, 2) Methodological quality (Risk of bias), 3) Clinical outcomes in RCTs, 4) Conclusions (Overall study conclusions and conclusions on reported clinical outcomes) and 5) Association between 
source of funding and methodological quality, clinical outcomes and author's conclusions.

\section{Search methods for identification of studies}

A literature search regardless of language was conducted on electronic databases including The Cochrane CENTRAL Register for Controlled Trials (2012), EMBASE (1980+), Scopus (1980 to 2012), EBSCO host (1960 to 2012), PUBMED / MEDLINE (1966 to 2012), OVID (1950 to 2012), SPORTDiscus (1960 to 2012), Web of Science (1970 to 2012), Science Direct (1950 to 2012), CINAHL (1980 to 2012), Science citation index (1970 to 2012), Latin American Caribbean Health Sciences literature (LILACS) (1965 to 2012), NLMGateway (1950-1966). RCTs published in nonEnglish language journals were translated by independent translators who were familiar with the subject matter.

The search strategy used to search PUBMED for studies on full term infants is: (synbiotic* and probiotic* OR prebiotic*) AND (FOS or fructooligosaccharide or inulin or GOS or galactooligosaccharide) AND (infant formula* OR infant feeding OR formula OR formula milk) AND (infant* or baby or babies) NOT (preterm or premature or low birth weight babies or allergy or eczema) AND (randomized controlled trial* OR controlled clinical trial* OR random allocation*) Limits: Humans. This search strategy was modified to search other electronic databases and for studies on preterm infants.

A hand search was conducted on abstracts of major conference proceedings such as the Pediatric Academic Society meetings from 1990 (www.pas-meetings.org), cross checked references cited in RCTs and in recent reviews (published from 2003 to 2012) for additional RCTs not identified by electronic searches and speciality journals which were not included in any database such as Pediatrika and Chinese Journal of Microecology. To identify on-going and unpublished trials, experts in the field, manufacturers of infant formula containing probiotics and prebiotics were contacted. Web sites of companies that have conducted or were conducting RCTs on probiotics and prebiotics were searched.

Examples: Pfizer (www.pfizerpro.com/clinicaltrials), Chris Hansen Laboratory (www.chr-hansen.com/research_ development/documentation.html). A search was conducted on prospective trial registries such as World Health Organization (WHO) International Clinical Trials Registry Platform Search Portal (www.who.int/trialsearch), Clinical Trials.gov register (www.clinicaltrials.gov), Current Controlled Trials metaRegister of Controlled Trials $[m R C T]$ (www.controlled-trials.com/mrct) and www.clinicaltrialresults.org.

\section{Selection of studies}

One reviewer (MM) independently reviewed all abstracts, citations and identified potentially eligible RCTs. The full reports of eligible RCTs were retrieved by one reviewer (MM) and the pre-specified selection criteria applied independently by two reviewers (MM, ML) using a study eligibility form designed for this review. If more than one publication of a study existed, all reports of the study were grouped together under one name. Any disagreements between the reviewers were resolved through discussion. Unresolved disagreements were resolved by a third party $(\mathrm{RB})$.

\section{Data extraction and management}

Two reviewers (MM, ML) independently extracted data using a pretested data extraction form that was designed for this review. The reviewers (MM, ML) cross checked data and resolved any differences through discussion. Unresolved disagreements were resolved by a third party (RB). One reviewer (MM) entered the data in SPSS version 19 and the other reviewer (AM) conducted quality control checks. The data obtained from each RCT included:

\section{A) Source of funding or support of RCTs}

The source of funding or support of the RCTs was defined and categorized as:

1) Industry included:

- For - profit company, donation of study product by a for - profit company which manufactured the study product,

- Not - for profit company that promoted the consumption of synbiotics, probiotics or prebiotics,

- Mixed sources (for-profit company and other source).

2) Non - industry included:

- Government: National, regional (provincial, county) government body with NO industry association.

- Foundation / Philanthropies: examples include Rockefeller foundation, Bill and Melinda Gates foundation.

- Institution: University, Research centres, teaching and academic hospitals.

- Other source of funding.

3) None: No source of funding was disclosed in study report.

\section{B) Assessment of methodological quality of evidence (Risk of bias)}

Two reviewers (MM, ML) independently assessed the risk of bias of included RCTs as described in the Cochrane Handbook for Systematic Reviews for Interventions according to the following 6 components: 1) sequence generation; 2) allocation concealment; 3 ) 
Table 1 Included studies and on-going studies

\begin{tabular}{|c|c|c|c|c|c|c|c|}
\hline \multicolumn{6}{|c|}{ Included studies } & \multicolumn{2}{|c|}{ On-going studies } \\
\hline Author publication year & $\begin{array}{l}\text { Full term/Preterm } \\
\text { infant }\end{array}$ & Sponsor & Author publication year & $\begin{array}{l}\text { Full term/Preterm } \\
\text { infant }\end{array}$ & Sponsor & $\begin{array}{l}\text { Author, Year } \\
\text { study commenced }\end{array}$ & $\begin{array}{c}\text { Full term/Preterm } \\
\text { infant }\end{array}$ \\
\hline Allen 2010 [18] & Full Term & $\begin{array}{l}\text { Knowledge exploitation } \\
\text { fund, collaborative } \\
\text { industrial research, others }\end{array}$ & Soh 2009 [19] & Full Term & $\begin{array}{l}\text { National Medical } \\
\text { Research Council, } \\
\text { Singapore }\end{array}$ & Jacobs 2007 [20] & Pre-Term \\
\hline $\begin{array}{l}\text { Alliet } 2007 \text { [21] } \\
\text { Scholtens } 2008 \text { [22] }\end{array}$ & Full Term & Numico & Urban 2008 [23] & Full Term & Nestle & Patole 2009 [24] & Pre-Term \\
\hline Ashley 2012 [25] & Full Term & Mead Johnson & Velaphi 2008 [26] & Full Term & Nestle & Underwood 2009 [27] & Pre-Term \\
\hline $\begin{array}{l}\text { Bakker-Zierikzee } 2005 \text { [28] } \\
\text { Bakker-Zierikzee } 2006 \text { [29] }\end{array}$ & Full Term & None/Not clear & Vendt 2006 [30] & Full Term & Valio Ltd & & \\
\hline Bettler 2006 [31] & Full Term & Wyeth Nutrition & Vlieger 2009 [32] & Full Term & Friesland & & \\
\hline Brunser 2006 [33] & Full Term & None/Not clear & Weizman 2005 [34] & Full Term & Materna Laboratories & & \\
\hline Bruzzese 2009 [35] & Full Term & Numico & Weizman 2006 [36] & Full Term & Marterna Laboratories & & \\
\hline Chouraqui 2004 [37] & Full Term & Nestle & Xiao-Ming 2004 [38] & Full Term & Friesland & & \\
\hline Chouraqui 2008 [39] & Full Term & Nestle & Xiao-Ming 2008 [40] & Full Term & None / Not clear & & \\
\hline Copper 2010 [41] & Full Term & Nestle & Ziegler 2007 [42] & Full Term & Mead Johnson & & \\
\hline Costalos 2008 [43] & Full Term & Numico & Bin-Nun 2005 [44] & Pre-Term & $\begin{array}{l}\text { Mr and Mrs Stephen } \\
\text { Hammerman, Mirsky } \\
\text { Research fund }\end{array}$ & & \\
\hline Decsi 2005 [45] & Full Term & Numil Ltd & $\begin{array}{l}\text { Boehm } 2002[46] \\
\text { Boehm } 2003[47] \\
\text { Knol } 2005[48]\end{array}$ & Pre-Term & Numico & & \\
\hline Fanaro 2005 [49] & Full Term & None / Not clear & Chrzanowska-Liszewska 2012 [50] & Pre-Term & None/Not clear & & \\
\hline Fanaro 2008 [51] & Full Term & Humana GmbH & Costalos 2003 [52] & Pre-Term & None/Not clear & & \\
\hline Gibson 2009 [53] & Full Term & Nestle & Dani 2002 [54] & Pre-Term & None/Not clear & & \\
\hline Gil-Campos 2012 [55] & Full Term & Puleva & Indrio 2008 [56] & Pre-Term & Bio Gaia & & \\
\hline Hascoet 2011 [57] & Full Term & Nestle & Indrio 2009 [58] & Pre-Term & Numico & & \\
\hline Holscher 2012a [59] & Full Term & Nestle & Kapiki 2007 [60] & Pre-Term & None/Not clear & & \\
\hline Holscher 2012b [61] & Full Term & Nestle & Kitajima 1992 [62] & Pre-Term & None/Not clear & & \\
\hline Kim 2010 [63] & Full Term & $\begin{array}{l}\text { Ministry of Health, Welfare } \\
\text { and family affairs. Republic } \\
\text { of Korea }\end{array}$ & Lin H-C 2008 [64] & Pre-Term & $\begin{array}{l}\text { National Science } \\
\text { Council of Taiwan }\end{array}$ & & \\
\hline Knol 2005 [65] & Full Term & Numico & Mihatsch 2006 [66] & Pre-Term & Milupa GmbH & & \\
\hline Magne 2008 [67] & Full Term & Numico & Mihatsch 2010 [68] & Pre-Term & Nestle & & \\
\hline Mah 2007 [69] & Full Term & $\begin{array}{l}\text { National Medical Research } \\
\text { Council Singapore }\end{array}$ & $\begin{array}{l}\text { Millar } 1993 \text { [70] } \\
\text { Stansbridge } 1993 \text { [71] }\end{array}$ & Pre-Term & $\begin{array}{l}\text { Wessex Regional Health } \\
\text { Authority and childrens } \\
\text { Research fund }\end{array}$ & & \\
\hline Maldonado 2010 [72] & Full Term & Puleva & Modi 2010 [73] & Pre-Term & Danone & & \\
\hline
\end{tabular}


Table 1 Included studies and on-going studies (Continued)

\begin{tabular}{|c|c|c|c|c|c|}
\hline $\begin{array}{l}\text { Moro } 2002[74] \\
\text { Moro } 2003[75]\end{array}$ & Full Term & None/Not clear & Mohan 2006 [76] & Pre-Term & None/Not clear \\
\hline Moro 2005 [77] & Full Term & None/Not clear & Reuman1986 [78] & Pre-Term & None/Not clear \\
\hline $\begin{array}{l}\text { Moro } 2006 \text { [79] } \\
\text { Arslanoglu } 2007 \text { [80] } \\
\text { Arslanoglu } 2008 \text { [81] } \\
\text { Van Hoffen } 2009 \text { [82] } \\
\text { Schouten } 2011 \text { [83] }\end{array}$ & Full Term & Numico & Riskin 2009 [84] & Pre-Term & None/Not clear \\
\hline Piemontese 2011 [85] & Full Term & Danone & Rouge 2009 [86] & Pre-Term & French Ministry of Health \\
\hline Puccio 2007 [87] & Full Term & Nestle & Sari 2011 [88] & Pre-Term & None/Not clear \\
\hline $\begin{array}{l}\text { Rautava } 2006 \text { [89] } \\
\text { Rautava } 2009 \text { [90] }\end{array}$ & Full Term & $\begin{array}{l}\text { Microbes and Man Research } \\
\text { program, Academy of Finland, } \\
\text { others }\end{array}$ & Stratiki 2007 [91] & Pre-Term & Nestle \\
\hline Rinne 2005 [92] & Full Term & $\begin{array}{l}\text { Academy of Finland, Turku } \\
\text { University Central Hospital } \\
\text { Research Funds }\end{array}$ & $\begin{array}{l}\text { Westerbeek } 2010 \text { [93] } \\
\text { Westerbeek 2011a [94] } \\
\text { Westerbeek 2011b [95] }\end{array}$ & Pre-Term & Danone \\
\hline Saavedra 2004 [96] & Full Term & Nestle & Yong 2009 [97] & Pre-Term & None/Not clear \\
\hline Scalabrin 2009 [98] & Full Term & Mead Johnson & & & \\
\hline Scalabrin 2012 [99] & Full Term & Mead Johnson & & & \\
\hline Schmelzle 2003 [100] & Full Term & Numico & & & \\
\hline
\end{tabular}




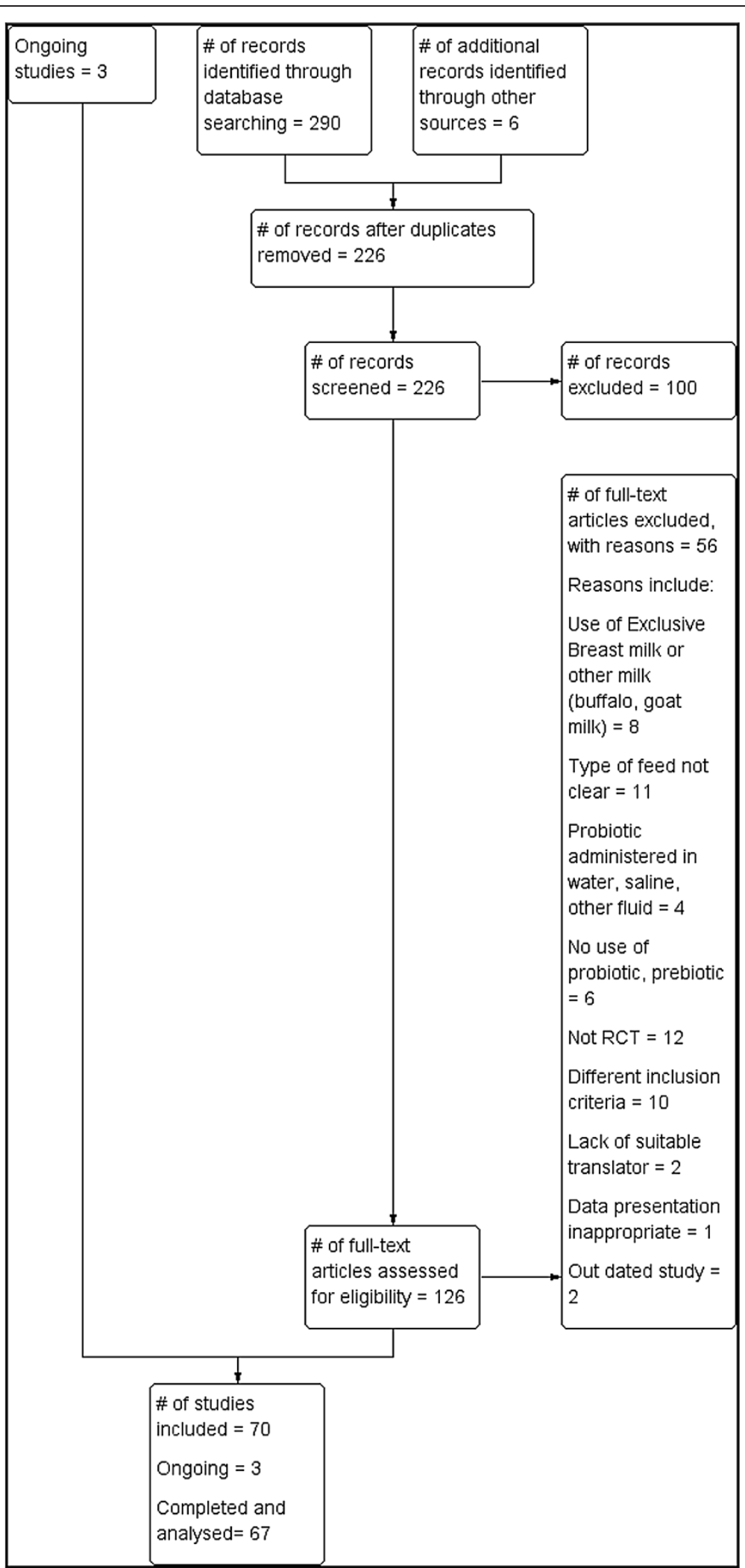

Figure 1 Process of study selection. 
Table 2 Table of $\mathbf{5 6}$ Excluded studies with reasons for exclusion

\begin{tabular}{|c|c|c|c|c|c|c|c|c|}
\hline $\begin{array}{l}\text { Use of Exclusive } \\
\text { breast milk or Other } \\
\text { milk feeds (buffalo, } \\
\text { goat milk) }\end{array}$ & $\begin{array}{l}\text { Type of feed } \\
\text { not clear/specified }\end{array}$ & $\begin{array}{l}\text { Probiotic administered } \\
\text { in water, saline or } \\
\text { other fluid that is } \\
\text { not infant formula }\end{array}$ & $\begin{array}{l}\text { No use of probiotic, } \\
\text { prebiotic }\end{array}$ & $\begin{array}{l}\text { Not RCT, (Cross } \\
\text { over, Follow up, } \\
\text { Observational study) }\end{array}$ & $\begin{array}{l}\text { Different inclusion } \\
\text { criteria }\end{array}$ & $\begin{array}{l}\text { Lack of suitable/ } \\
\text { knowledgeable } \\
\text { translator }\end{array}$ & $\begin{array}{l}\text { Data presentation } \\
\text { inappropriate }\end{array}$ & $\begin{array}{l}\text { Out dated } \\
\text { (published } \\
\text { before 1980) }\end{array}$ \\
\hline Agarwal 2003 [101] & Al Hosni 2012 [102] & FengJuan 2008 [103] & Morisset 2011 [104] & Huet 2006 [105] & Agustina 2007 [106] & $\begin{array}{l}\text { Akiyama1994a [107] } \\
\text { (Japanese) }\end{array}$ & Grzéskowak 2012 [108] & Andrews 1969 [109] \\
\hline Baldeon 2008 [110] & Campeotto 2011 [111] & Kuitunen 2009 [112] & Patole 2005 [113] & Bongers 2007 [114] & Correa 2005 [115] & $\begin{array}{l}\text { Akiyama1994b [116] } \\
\text { (Japanese) }\end{array}$ & & Robinson 1952 [117] \\
\hline Braga 2011 [118] & Cukrowska 2002 [119] & Kukkonen 2007 [120] & Rochat 2007 [121] & Chou I-C 2009 [122] & Hol 2008 [123] & & & \\
\hline Chandra 2002 [124] & *Karvonen 1999 [125] & Kukkonen 2008 [126] & Taipale 2011 [127] & Euler 2005 [128] & Isolauri 2000 [129] & & & \\
\hline Lin H-C 2005 [130] & *Karvonen 2001 [131] & & Taylor 2009 [132] & Hoyos 1999 [133] & Nopchinda 2002 [134] & & & \\
\hline Manzoni 2006 [135] & *Karvonen 2002 [136] & & Thibault 2004 [137] & Kim 2007 [138] & Rivero 2004 [139] & & & \\
\hline Rinne 2006 [140] & Li 2004 [141] & & & Lee 2007 [142] & Urao 1999 [143] & & & \\
\hline \multirow[t]{5}{*}{ Samanta 2009 [144] } & Panigrahi 2008 [145] & & & Lidesteri 2003 [146] & Van der Aa 2010 [147] & & & \\
\hline & Rojas 2012 [148] & & & Marini 2003 [149] & Waliogora-Dupriet 2007 [150] & & & \\
\hline & Taylor 2007 [151] & & & Rigo 2001 [152] & Wang 2007 [153] & & & \\
\hline & Underwood 2009 [154] & & & Savino 2003 [155] & & & & \\
\hline & & & & Sepp 1993 [156] & & & & \\
\hline
\end{tabular}

Key: * Unpublished trials. 
blinding; 4) incomplete outcome data; 5) selective outcome reporting; and 6) other sources of bias [17]. Each domain was assessed as having either a low risk of bias, high risk of bias or unclear to permit judgment. Any disagreements regarding risk of bias were resolved through discussion between MM, ML and RB. The association between risk of bias (domains) and type of funding (industry, non - industry, none declared) was explored.

\section{C) Assessment of clinical outcomes}

The primary and secondary outcomes from each study report were evaluated and categorized as:

1. Positive: synbiotic, probiotic or prebiotic supplementation had a statistically significant effect, $\mathrm{p}<0.05$.

Examples of positive outcomes included: adequate growth (weight gain, length gain, head circumference), tolerance (no feeding problems), microflora (increase in colony forming units of bifidobacteria, lactobacillus, decrease in pathogens), decreased infections (decrease in frequency, incidence of infections).

2) Negative: synbiotic, probiotic or prebiotic supplementation had a statistically significant effect in an adverse event / negative outcome such as weight loss, diarrhoea, $\mathrm{p}<0.05$

3) Neutral: synbiotic, probiotic or prebiotic supplementation did not have a statistically significant effect, $\mathrm{p}>0.05$, no significant differences between study groups. Clinical outcomes included: growth parameters, gastrointestinal parameters (tolerance to feed, stool characteristics, microflora); immune response, infections and mortality.

\section{D) Overall study conclusions and conclusions on reported outcomes}

The authors' overall study conclusion and conclusions on reported clinical outcomes were evaluated and categorized as:

1. Positive: The author's conclusion preferred the sponsor's products over control/placebo. Interpretation of data supported the sponsor's products over control.

2. Negative: The sponsors' products were not preferred over control / placebo. Interpretation of data did NOT support the sponsors' products.

3. Neutral: The author's conclusion was neutral to the sponsor's products.

4. No clear conclusion was offered by author.

In this review, the "conclusions on reported outcomes" referred to the authors' conclusions on individual reported
Table 3 Source of funding and study participants

\begin{tabular}{|c|c|c|c|}
\hline \multirow[b]{2}{*}{ Sponsor } & \multicolumn{2}{|c|}{ Study participants } & \multirow{3}{*}{$\begin{array}{l}\text { Total } \\
\text { n (\%) }\end{array}$} \\
\hline & Full term infant & Preterm Infant & \\
\hline & $\mathrm{n}$ & $\mathrm{n}$ & \\
\hline Industry & 33 & 7 & $40(59.7)$ \\
\hline None / Not Clear & 6 & 10 & $16(23.9)$ \\
\hline Non Industry & 6 & 5 & $11(16.4)$ \\
\hline Total & 45 & 22 & $67(100.0$ \\
\hline
\end{tabular}

RCTs outcomes. Examples include conclusions on weight gain, length gain, vomiting, necrotizing enterocolitis, sepsis.

\section{Statistical Analysis}

All the outcomes in this review were dichotomous and are described in frequencies and percentages. The association between source of funding (industry/nonindustry/ none) and methodological quality (low/unclear/high risk of bias), clinical outcomes and author's conclusions were assessed using both the Pearson's Chisquare test and the Fisher's exact test. A p-value of less than 0.05 was considered statistically significant. SPSS version 19 statistical software was used. A statistician (AM) was consulted throughout the review process.

\section{Ethics}

The Human Research Ethics Committee at Stellenbosch University, South Africa reviewed the protocol for this review, ruled that all data to be collected for this review was from the public domain and was therefore exempt from ethical approval.

\section{Results}

Results of the search and description of studies

Electronic search of available databases yielded 290 citations. After reading titles and abstracts, duplicate reports were removed, 226 articles were screened and 100 articles were excluded. A hand search yielded 6 more articles. Potentially relevant full text reports were retrieved, reviewed for eligibility and a further 56 RCTs were excluded. Studies that had multiple publications were considered as one trial. Sixty seven RCTs and three on-going RCTs were

Table 4 Methodological quality (Risk of bias)

\begin{tabular}{llll}
\hline & \multicolumn{3}{c}{$\mathbf{N}(\%)$} \\
\cline { 2 - 4 } Quality of studies N=67 & Low risk & High risk & Unclear \\
\hline Sequence generation & $42(62.7)$ & & $25(37.3)$ \\
Allocation concealment & $32(47.8)$ & $35(52.2)$ \\
Blinding & $31(46.3)$ & $36(53.7)$ \\
Incomplete Outcome data & $52(77.6)$ & $1(1.5)$ & $14(20.9)$ \\
Selective reporting & $57(85.1)$ & $7(10.4)$ & $3(4.5)$ \\
Other bias & $53(79.1)$ & & $14(20.9)$ \\
\hline
\end{tabular}




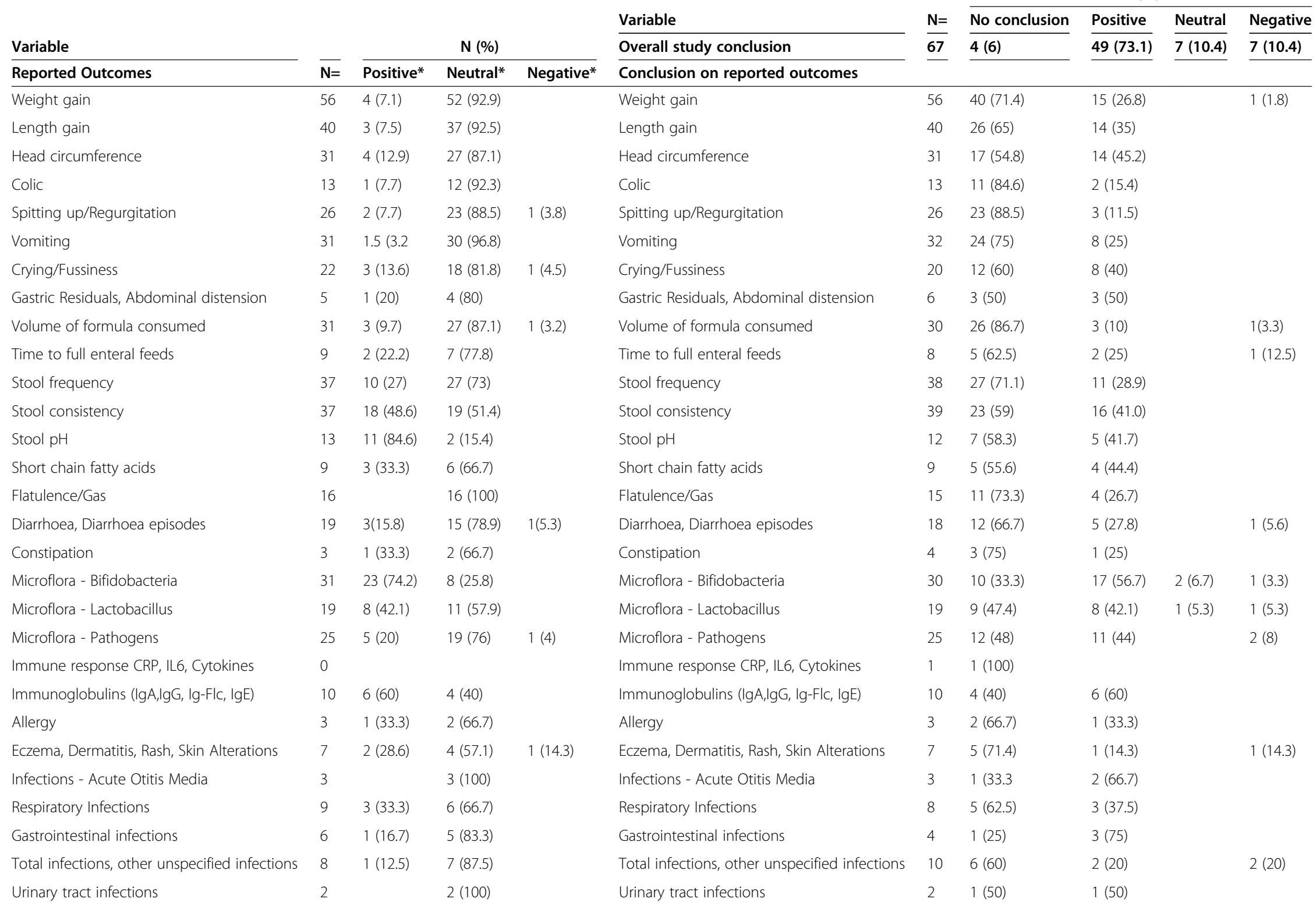


Table 5 Reported outcomes and conclusions (Continued)

\begin{tabular}{|c|c|c|c|c|c|c|c|c|}
\hline Necrotizing Enterocolitis & 11 & $2(18.2)$ & $9(81.8)$ & Necrotizing Enterocolitis & 12 & $7(58.3)$ & $3(25)$ & $2(16.7)$ \\
\hline Sepsis & 10 & & $10(100)$ & Sepsis & 10 & $9(90)$ & $1(10)$ & \\
\hline Fever, Febrile Episodes & 4 & $2(50)$ & $2(50)$ & Fever, Febrile Episodes & 2 & $2(100)$ & & \\
\hline Antibiotic use & 19 & $4(21.1)$ & $15(78.9)$ & Antibiotic use & 16 & $13(81.3)$ & $3(18.8)$ & \\
\hline Hospitalization & 12 & & $12(100)$ & Hospitalization & 10 & $10(100)$ & & \\
\hline Biochemical measures & 9 & & $9(100)$ & Biochemical measures & 6 & $5(83.3)$ & $1(16.7)$ & \\
\hline Adverse events & 18 & $2(11.1)$ & $16(88.9)$ & Adverse events & 17 & $13(76.5)$ & $4(23.5)$ & \\
\hline Death / Mortality & 7 & $1(14.3$ & $6(85.7)$ & Death/Mortality & 8 & $7(87.5)$ & $1(12.5)$ & \\
\hline Intestinal permeability & 3 & $1(33.3)$ & $2(66.7)$ & Intestinal permeability & 3 & $1(33.3)$ & $2(66.7)$ & \\
\hline Duration of TPN & 5 & & $5(100)$ & Duration of TPN & 5 & $4(80)$ & $1(20)$ & \\
\hline
\end{tabular}

*Positive: synbiotic, probiotic or prebiotic supplementation had a statistically significant effect, $\mathrm{p}<0.05$.

*Neutral: synbiotic, probiotic or prebiotic supplementation did not have a statistically significant effect, $p>0.05$

*Negative: synbiotic, probiotic or prebiotic supplementation had a statistically significant increase in an adverse event / negative outcome, $p<0.05$. 
Table 6 Association between Sponsor and methodological quality (risk of bias)

\begin{tabular}{|c|c|c|c|c|c|c|}
\hline $\begin{array}{l}\text { Methodological quality } \\
\mathrm{N}=67 \text { studies }\end{array}$ & Source of funding & $\frac{\text { Yes (Low risk) }}{n(\%)^{\$ \$}}$ & $\frac{\text { No (High risk) }}{n(\%)^{\$ \$}}$ & $\frac{\text { Unclear }}{\mathrm{n}(\%)^{\$ \$}}$ & $\begin{array}{l}\text { Chi-square } \\
\text { p value }\end{array}$ & $\begin{array}{c}\text { Fisher's exact } \\
\text { p value }\end{array}$ \\
\hline \multirow[t]{3}{*}{ Sequence generation } & Industry & $26(38.8)$ & & $14(20.9)$ & 0.435 & 0.465 \\
\hline & None/Not clear & $8(11.9)$ & & $8(11.9)$ & & \\
\hline & Non industry & $8(11.9)$ & & $3(4.5)$ & & \\
\hline \multirow[t]{3}{*}{ Allocation concealment } & Industry & $21(31.3)$ & & 19 (28.4) & 0.315 & 0.338 \\
\hline & None/Not clear & $5(7.5)$ & & $11(16.4)$ & & \\
\hline & Non lindustry & $6(9.0)$ & & $5(7.5)$ & & \\
\hline \multirow[t]{3}{*}{ Blinding } & Industry & $18(26.9)$ & & $22(32.8)$ & 0.395 & 0.457 \\
\hline & None/Not clear & $6(9.0)$ & & $10(14.9)$ & & \\
\hline & Non industry & $7(10.4)$ & & $4(6.0)$ & & \\
\hline \multirow[t]{3}{*}{ Incomplete outcome data } & Industry & $36(53.7)$ & $1(1.5)$ & $3(4.5)$ & $0.023^{*}$ & $0.005^{*}$ \\
\hline & None/Not clear & $9(13.4)$ & & $7(10.4)$ & & \\
\hline & Non industry & $7(10.4)$ & & $4(6.0)$ & & \\
\hline \multirow[t]{3}{*}{ Selective reporting } & Industry & $36(53.7)$ & $2(3.0)$ & $2(3.0)$ & 0.224 & 0.188 \\
\hline & None/Not clear & $11(16.4)$ & $4(6.0)$ & $1(1.5)$ & & \\
\hline & Non industry & $10(14.9)$ & $1(1.5)$ & 0 & & \\
\hline \multirow[t]{3}{*}{ Free of other bias } & Industry & $35(52.2)$ & & $5(7.5)$ & $0.033^{*}$ & $0.038^{*}$ \\
\hline & None/Not clear & $9(13.4)$ & & $7(10.4)$ & & \\
\hline & Non industry & $8(13.4)$ & $1(1.5)$ & $2(3.0)$ & & \\
\hline
\end{tabular}

*Significant $p<0.05$.

$\$$ Overall percentage.

included in this review. (Table 1) The selection process is shown in Figure 1. Table 2 gives a list of 56 RCTs which were excluded for: use of exclusive breast or non-formula milk (8 RCTs), type of feed not clear (11 RCTs), probiotic administered in saline, water or other fluid (4 RCTs), no use of probiotic or prebiotic (6 RCTs), not RCT (12 studies), different inclusion criteria (10 studies), lack of suitable translator $(2 \mathrm{RCTs})$, data presentation inappropriate
(1 RCT) and out of date [published before1980] (2 RCTs). Three excluded RCTs were unpublished trials.

\section{Characteristics of included studies}

Table 1 lists included and on-going trials. Sixty seven RCTs were included, $45(67.2 \%)$ on full term infants, 22 (32.8\%) on preterm infants. All included RCTs were published trials. All trials were conducted on healthy full

Table 7 Association between Sponsor and clinical outcomes: Growth

\begin{tabular}{|c|c|c|c|c|c|}
\hline \multirow{3}{*}{ Growth } & \multirow{3}{*}{ Source of funding } & \multicolumn{2}{|c|}{ Assessment of outcome } & \multirow{3}{*}{ Chi-square $p$ value } & \multirow{3}{*}{ Fisher's exact $p$ value } \\
\hline & & Positive* & Neutral* & & \\
\hline & & $n(\%)^{\$ \$}$ & $n(\%)^{\$ \$}$ & & \\
\hline \multirow[t]{3}{*}{ Weight gain $N=56$} & Industry & $2(3.6)$ & $35(62.5)$ & 0.309 & 0.266 \\
\hline & None/Not clear & $2(3.6)$ & $10(17.9)$ & & \\
\hline & Non industry & 0 & $7(12.5)$ & & \\
\hline \multirow[t]{3}{*}{ Length gain $N=40$} & Industry & $3(7.5)$ & $29(72.5)$ & 0.667 & 1.00 \\
\hline & None/Not clear & & $6(15)$ & & \\
\hline & Non industry & & $2(5)$ & & \\
\hline \multirow[t]{3}{*}{ Head Circumference $N=31$} & Industry & $4(12.9)$ & $23(74.2)$ & 0.712 & 1.00 \\
\hline & None /Not clear & & $3(9.7)$ & & \\
\hline & Non industry & & $1(3.2)$ & & \\
\hline
\end{tabular}

\$Overall percentage.

*Positive: synbiotic, probiotic or prebiotic supplementation had a statistically significant effect, $p<0.05$. There were significant differences between study groups (in favour of experimental group).

*Neutral: synbiotic, probiotic or prebiotic supplementation did not have a statistically significant effect, $p>0.05$, No significant differences between study groups. 
term or preterm infants and used standard (full term or preterm) infant formula (Table 3 ).

\section{Funding}

Out of 67 trials, 40 (59.7\%) were funded by food industry, $11(16.4 \%)$ were funded by non-industry entities, and $16(23.9 \%)$ did not specify their source of funding, 10 RCTs on preterm infants, 6 RCTs on full infants (Table 3).

\section{Methodological quality (Risk of bias)}

In this review, several domains were not adequately reported, particularly, the domains of sequence generation, allocation concealment and blinding. Out of 67 RCTs, $25(37.3 \%)$ failed to report sequence generation, 35 (52.2\%) failed to report allocation concealment and 36 (53.7\%) did not report blinding. Majority of the RCTs were assessed as having a low risk of bias in the domains of incomplete outcome data $52(77.6 \%)$, selective reporting 57(85.1\%) and other bias 53 (79.1\%) (Table 4).

\section{Outcomes and study conclusions}

In most RCTs, majority of outcomes were assessed as neutral, (intervention did not have a statistically significant effect, $\mathrm{p}>0.05)$. A total of $49(73.1 \%)$ of RCTs had a positive overall study conclusion in favour of the sponsors' products, while 7 (10.4\%) had negative, 7 (10.4\%) had neutral conclusions and 4 (6\%) had no clear conclusion. The included RCTs either did not provide any conclusion on their reported clinical outcomes or, they provided a positive conclusion for their reported outcome in-favour of the sponsors' products. Few RCTS had either negative or neutral conclusions on their reported clinical outcomes (Table 5).

\section{Association between source of funding (sponsor) and methodological quality of studies}

There was no significant association between the source of funding and the domains of sequence generation (Chi square $\mathrm{p}=0.435$, Fisher exact $\mathrm{p}=0.465$ ), allocation concealment (Chi - square $\mathrm{p}=0.315$, Fisher exact $\mathrm{p}=0.338$ ), blinding (Chi - square $\mathrm{p}=0.395$, Fisher exact $\mathrm{p}=0.457$ )

Table 8 Association between Sponsor and clinical outcomes: Tolerance symptoms

\begin{tabular}{|c|c|c|c|c|c|c|}
\hline Tolerance & Source of funding & $\frac{\text { Positive }^{*}}{\mathrm{n}(\%)^{\$ \$}}$ & $\frac{\text { Negative* }^{*}}{n(\%)^{\$ \$}}$ & $\frac{\text { Neutral }^{*}}{n(\%)^{\$ \$}}$ & $\begin{array}{l}\text { Chi-square } \\
\mathrm{p} \text { value }\end{array}$ & $\begin{array}{l}\text { Fisher's exact } \\
\text { p value }\end{array}$ \\
\hline \multirow[t]{3}{*}{ Colic N = 13} & Industry & $1(7.7)$ & & $11(84.6)$ & 0.764 & 1.00 \\
\hline & None/Not clear & & & & & \\
\hline & Non industry & & & $1(7.7)$ & & \\
\hline \multirow[t]{3}{*}{ Spitting up/Regurgitation $N=26$} & Industry & $2(7.7)$ & $1(3.8)$ & $17(65.4)$ & 0.907 & 1.00 \\
\hline & None/Not clear & & & $4(15.4)$ & & \\
\hline & Non industry & & & $2(7.7)$ & & \\
\hline \multirow[t]{3}{*}{ Vomiting $N=31$} & Industry & $1(3.2)$ & & $23(74.2)$ & 0.860 & 1.00 \\
\hline & None/Not clear & & & $5(16.1)$ & & \\
\hline & Non industry & & & $2(6.5)$ & & \\
\hline \multirow[t]{3}{*}{ Crying fussiness $\mathrm{N}=22$} & Industry & $3(13.6)$ & $1(4.5)$ & $14(63.6)$ & 0.581 & 1.00 \\
\hline & None/Not clear & & & $4(18.2)$ & & \\
\hline & Non industry & & & 0 & & \\
\hline \multirow[t]{3}{*}{ Gastric residuals, Abdominal distension N $=5$} & Industry & & & $1(20)$ & 0.659 & 1.00 \\
\hline & None/Not clear & & & $1(20)$ & & \\
\hline & Non industry & $1(6.7)$ & & $2(40)$ & & \\
\hline \multirow[t]{3}{*}{ Volume of formula consumed/daily intake $\mathrm{N}=31$} & Industry & $3(9.7)$ & $1(3.2)$ & $18(58.1)$ & 0.758 & 1.00 \\
\hline & None/Not clear & & & $4(12.9)$ & & \\
\hline & Non industry & & & $5(16.1)$ & & \\
\hline \multirow[t]{3}{*}{ Days to full enteral feeding $N=9$} & Industry & & & $4(44.4)$ & 0.325 & 0.444 \\
\hline & None/Not clear & $1(11.1)$ & & $1(11.1)$ & & \\
\hline & Non industry & $1(11.1)$ & & $2(22.2)$ & & \\
\hline
\end{tabular}

\footnotetext{
\$\$ Overall percentage.
}

*Positive: synbiotic, probiotic or prebiotic supplementation had a statistically significant effect, $\mathrm{p}<0.05$. There were significant differences between study groups (in favour of experimental group).

*Neutral: synbiotic, probiotic or prebiotic supplementation did not have a statistically significant effect, $p>0.05$, No significant differences between study groups.

*Negative: synbiotic, probiotic or prebiotic supplementation had a statistically significant increase in an adverse event / negative outcome, $\mathrm{p}<0.05$. 
Table 9 Association between sponsor and clinical outcomes: stool characteristics

\begin{tabular}{|c|c|c|c|c|c|c|}
\hline Stool characteristics & Source of funding & $\frac{\text { Positive }^{*}}{\text { n (\%) }}$ & $\frac{\text { Negative* }^{*}}{n(\%)^{\$ \$}}$ & $\frac{\text { Neutral }^{*}}{\mathrm{n}(\%)^{\$ \$}}$ & $\begin{array}{l}\text { Chi-square } \\
\mathrm{p} \text { value }\end{array}$ & $\begin{array}{l}\text { Fisher's exact } \\
\text { p value }\end{array}$ \\
\hline \multirow[t]{3}{*}{ Stool Frequency N = 37} & Industry & $7(18.9)$ & & $22(59.5)$ & 0.501 & 0.540 \\
\hline & None/Not clear & $3(8.1)$ & & $4(10.8)$ & & \\
\hline & Non industry & & & $1(2.7)$ & & \\
\hline \multirow[t]{3}{*}{ Stool Consistency $n=37$} & Industry & $14(37.8)$ & & $15(40.5)$ & 0.562 & 1.00 \\
\hline & None/Not clear & $4(10.8)$ & & $3(8.1)$ & & \\
\hline & Non industry & & & $1(2.7)$ & & \\
\hline \multirow[t]{3}{*}{ Stool pH N =13 } & Industry & $7(53.8)$ & & $2(15.4)$ & 0.305 & 1.00 \\
\hline & None/Not clear & $4(30.8)$ & & & & \\
\hline & Non industry & & & & & \\
\hline \multirow[t]{3}{*}{ Stool Short Chain Fatty Acids $N=9$} & Industry & $2(22.2)$ & & $4(44.4)$ & 0.687 & 1.00 \\
\hline & None / Not clear & $1(11.1)$ & & $1(11.1)$ & & \\
\hline & Non industry & & & $1(11.1)$ & & \\
\hline \multirow[t]{3}{*}{ Flatulence / Gas N = 16} & Industry & & & $15(93.8)$ & Not valid & \\
\hline & None/Not clear & & & $1(6.3)$ & & \\
\hline & Non industry & & & 0 & & \\
\hline \multirow[t]{3}{*}{ Diarrhoea, Diarrhoea episodes N=19 } & Industry & $3(15.8)$ & $1(5.3)$ & $10(52.6)$ & 0.771 & 1.00 \\
\hline & None/Not clear & & & $2(10.5)$ & & \\
\hline & Non industry & & & $3(15.8)$ & & \\
\hline \multirow[t]{3}{*}{ Constipation $\mathrm{N}=3$} & Industry & $1(33.3)$ & & $1(33.3)$ & 0.386 & 1.00 \\
\hline & None/Not clear & & & $1(33.3)$ & & \\
\hline & Non industry & & & 0 & & \\
\hline
\end{tabular}

\$\$Overall percentage.

*Positive: synbiotic, probiotic or prebiotic supplementation had a statistically significant effect, $\mathrm{p}<0.05$. There were significant differences between study groups (in favour of experimental group).

*Neutral: synbiotic, probiotic or prebiotic supplementation did not have a statistically significant effect, $p>0.05$, No significant differences between study groups. *Negative: synbiotic, probiotic or prebiotic supplementation had a statistically significant increase in an adverse event / negative outcome, $\mathrm{p}<0.05$.

Table 10 Association between sponsor and clinical outcomes: Microflora

\begin{tabular}{|c|c|c|c|c|c|c|}
\hline Microflora & Source of funding & $\frac{\text { Positive } 4^{*}}{\mathrm{n}(\%)^{\$ \$}}$ & $\frac{\text { Negative } 5^{*}}{\mathrm{n}(\%)^{\$ \$}}$ & $\frac{\text { Neutral } 6^{*}}{\mathrm{n}(\%)^{\$ \$}}$ & $\begin{array}{l}\text { Chi-square } \\
\text { p value }\end{array}$ & $\begin{array}{l}\text { Fisher's exact } \\
\text { p value }\end{array}$ \\
\hline \multirow[t]{3}{*}{ Bifidobacteria $N=31$} & Industry & $12(38.7)$ & & $6(19.4)$ & 0.416 & 0.583 \\
\hline & None/Not clear & $8(25.8)$ & & $2(6.5)$ & & \\
\hline & Non industry & $3(9.7)$ & & & & \\
\hline \multirow[t]{3}{*}{ Lactobacillus N = 19} & Industry & $2(10.5)$ & & $6(31.6)$ & 0.155 & 0.176 \\
\hline & None/Not clear & $4(21.1)$ & & $5(26.3)$ & & \\
\hline & Non industry & $2(10.5)$ & & 0 & & \\
\hline \multirow[t]{3}{*}{ Pathogens $N=25$} & Industry & $2(8.0)$ & & $11(44.0)$ & 0.532 & 0.612 \\
\hline & None/Not clear & $3(12.0)$ & $1(4.0)$ & $6(24.0)$ & & \\
\hline & Non industry & & & $2(8.0)$ & & \\
\hline
\end{tabular}

\$\$ Overall percentage.

*Positive: synbiotic, probiotic or prebiotic supplementation had a statistically significant effect, $p<0.05$. There were significant differences between study groups (in favour of experimental group).

*Neutral: synbiotic, probiotic or prebiotic supplementation did not have a statistically significant effect, $p>0.05$, No significant differences between study groups.

*Negative: synbiotic, probiotic or prebiotic supplementation had a statistically significant increase in an adverse event / negative outcome, $p<0.05$. 
Table 11 Association between sponsor and clinical outcomes: Necrotizing enterocolitis, sepsis and antibiotic use

\begin{tabular}{|c|c|c|c|c|c|}
\hline & Source of funding & $\frac{\text { Positive }^{*}}{\mathrm{n}(\%)^{\$ \$}}$ & $\frac{\text { Neutral* }}{n(\%)^{\$ \$}}$ & $\begin{array}{l}\text { Chi-square } \\
p \text { value }\end{array}$ & $\begin{array}{l}\text { Fisher's exact } \\
\text { p value }\end{array}$ \\
\hline \multirow[t]{3}{*}{ Necrotising enterocolitis $N=11$} & Industry & & $4(36.4)$ & 0.118 & 0.273 \\
\hline & None/Not clear & & $3(27.3)$ & & \\
\hline & Non industry & $2(18.2)$ & $2(18.2)$ & & \\
\hline \multirow[t]{3}{*}{ Sepsis $N=10$} & Industry & & $2(20)$ & Not Valid & \\
\hline & None/Not clear & & $3(30)$ & & \\
\hline & Non industry & & $5(50)$ & & \\
\hline \multirow[t]{3}{*}{ Antibiotic use $\mathrm{N}=19$} & Industry & $4(21.1)$ & $4(21.1)$ & $0.031^{\#}$ & $0.039^{\#}$ \\
\hline & None/Not clear & & $5(26.3)$ & & \\
\hline & Non industry & & $6(31.6)$ & & \\
\hline
\end{tabular}

\$ Overall percentage.

*Positive: synbiotic, probiotic or prebiotic supplementation had a statistically significant effect, $p<0.05$. There were significant differences between study groups (in favour of experimental group).

*Neutral: synbiotic, probiotic or prebiotic supplementation did not have a statistically significant effect, $p>0.05$, No significant differences between study groups.

\# Significant $\mathrm{p}<0.05$

and selective reporting (Chi - square $\mathrm{p}=0.224$, Fisher exact $\mathrm{p}=0.188$ ) (Table 6).

There was a significant association between funding and the domains of incomplete outcome data (Chi square $\mathrm{p}=0.023$, Fisher exact $\mathrm{p}=0.005$ ) and free of other bias (Chi - square $\mathrm{p}=0.033$, Fisher exact $\mathrm{p}=$ 0.038) (Table 6). The association between source of funding and incomplete outcome data was such that industry-funded trials had significantly less missing data than non-industry funded trials. The association between source of funding and free of other bias (such as outcomes bias) was such that a significantly higher percentage of industry-funded trials were free of other bias compared to non-industry-funded trials.

\section{Association between source of funding (sponsor) and clinical outcomes}

There was no significant association between source of funding and reporting of clinical outcomes: Growth parameters, stool characteristics, microflora, infections (Tables 7, 8, 9, 10 and 11), immune parameters, adverse events and mortality (data not shown). There was a significant association between the source of funding and reporting of antibiotic use in formula fed infants (Chi-square $\mathrm{p}=0.031$, Fisher exact $\mathrm{p}=0.039$ ) such that industry funded trials were more likely to decrease the use of antibiotics than non-industry funded trials (Table 11).

\section{Association between source of funding (sponsor) and overall study conclusion}

There was no significant association between sources of funding and overall study conclusion (Chi-square $\mathrm{p}=$ 0.505 , Fisher exact $p=0.373)$. Majority of RCTs, 49 (73.1\%), had a positive study conclusion; $32(47.8 \%)$ of these RCTs, were industry sponsored, 7 (10.4\%) non- industry and $10(14.9 \%)$ which did not declare their source of funding (Table 12). A sensitivity analysis was conducted with respect to combining industry sponsored studies with those that had not declared their source of funding. There was no change in the results. There was no significant association between source of funding and overall study conclusion (Chi-square $\mathrm{p}=0.483$, Fisher exact $\mathrm{p}=0.425$ ).

\section{Association between source of funding (sponsor) and conclusion on reported clinical outcomes}

There was a significant association between source of funding and conclusion on weight gain (Chi-square $\mathrm{p}=$ 0.037 , Fisher exact $p=0.024$ ) such that industry-funded

Table 12 Association between sponsor and OVERALL study conclusion

\begin{tabular}{|c|c|c|c|c|c|c|c|c|}
\hline & \multirow[t]{2}{*}{ Source of funding } & \multirow{2}{*}{$\frac{\text { Positive }}{\text { n (\%) }}$} & \multirow{2}{*}{$\frac{\text { Negative }}{n(\%)^{\$ \$}}$} & \multirow{2}{*}{$\frac{\text { Neutral }}{n(\%)^{\$ \$}}$} & \multirow{2}{*}{$\frac{\text { No clear conclusion }}{n(\%)^{\$ \$}}$} & \multirow{2}{*}{$\frac{\text { Total }}{\text { n }(\%)^{\$ \$}}$} & \multirow{2}{*}{$\begin{array}{l}\text { Chi-square } \\
\text { p value }\end{array}$} & \multirow{2}{*}{$\begin{array}{c}\text { Fisher's exac } \\
\text { p value }\end{array}$} \\
\hline & & & & & & & & \\
\hline \multirow[t]{4}{*}{ Overall conclusion $N=67$} & Industry & $32(47.8)$ & $2(3.0)$ & $3(4.5)$ & $3(4.5)$ & $40(59.7 \%)$ & 0.505 & 0.373 \\
\hline & None/Not clear & $10(14.9)$ & $3(4.5)$ & $2(3.0)$ & $1(1.5)$ & $16(23.9 \%)$ & & \\
\hline & Non industry & $7(10.4)$ & $2(3.0)$ & $2(3.0)$ & 0 & $11(16.4 \%)$ & & \\
\hline & Total & $49(73.1 \%)$ & $7(10.4 \%)$ & $7(10.4 \%)$ & $4(6.0 \%)$ & $67(100)$ & & \\
\hline
\end{tabular}

\$\$ Overall percentage. 
Table 13 Association between sponsor and conclusion on reported outcome: Growth parameters

\begin{tabular}{|c|c|c|c|c|c|c|}
\hline \multirow[t]{2}{*}{ Authors conclusion on: } & \multirow[t]{2}{*}{ Source of funding } & \multirow{2}{*}{$\frac{\text { No conclusion on reported outcome }}{n(\%)^{\$ \$}}$} & \multirow{2}{*}{$\frac{\text { Positive }}{\mathrm{n}(\%)^{\$ \$}}$} & \multirow{2}{*}{$\frac{\text { Negative }}{n(\%)^{\$ \$}}$} & \multirow{2}{*}{$\begin{array}{l}\text { Chi-square } \\
\text { p value }\end{array}$} & \multirow{2}{*}{$\begin{array}{c}\text { Fisher's exact } \\
\text { p value }\end{array}$} \\
\hline & & & & & & \\
\hline \multirow[t]{3}{*}{ Weight gain $N=56$} & Industry & $23(41.1 \%)$ & $14(25.0 \%)$ & & $0.037^{\#}$ & $0.024^{\#}$ \\
\hline & None/Not clear & $10(17.9 \%)$ & $1(1.8 \%)$ & $1(1.8 \%)$ & & \\
\hline & Non industry & $7(12.5 \%)$ & & & & \\
\hline \multirow[t]{3}{*}{ Length gain $N=40$} & Industry & $18(45 \%)$ & $14(35 \%)$ & & 0.068 & 0.051 \\
\hline & None/Not clear & $6(15 \%)$ & & & & \\
\hline & Non industry & $2(5)$ & & & & \\
\hline \multirow[t]{3}{*}{ Head circumference $N=31$} & Industry & $13(41.9)$ & $14(45.2)$ & & 0.151 & 0.232 \\
\hline & None/Not clear & $3(9.7)$ & & & & \\
\hline & Non industry & $1(3.2)$ & & & & \\
\hline
\end{tabular}

${ }^{\#}$ Significant $\mathrm{p}<0.05,{ }^{\text {\$\$ }}$ Overall percentage.

trials were more likely to report positive conclusions on weight gain than non-industry-funded trials (Table 13). There was no significant association between source of funding and conclusion on other reported clinical outcomes (Tables 14, 15, 16 and 17).

\section{Discussion}

This review revealed that majority of RCTs (from 1980 to 2012) on infants fed formula supplemented with probiotics, prebiotics or synbiotics are funded by the food industry. This is consistent with the trend that

Table 14 Association between sponsor and conclusion on reported outcome: Tolerance symptoms

\begin{tabular}{|c|c|c|c|c|c|c|}
\hline \multirow[t]{2}{*}{ Tolerance } & \multirow[t]{2}{*}{ Source of funding } & No conclusion on reported outcome & \multirow{2}{*}{$\frac{\text { Positive }}{\mathrm{n}(\%)^{\$ \$}}$} & \multirow{2}{*}{$\frac{\text { Negative }}{n(\%)^{\$ \$}}$} & \multirow{2}{*}{$\begin{array}{l}\text { Chi-square } \\
\mathrm{p} \text { value }\end{array}$} & \multirow{2}{*}{$\begin{array}{l}\text { Fisher's exact } \\
\mathrm{p} \text { value }\end{array}$} \\
\hline & & 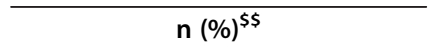 & & & & \\
\hline \multirow[t]{3}{*}{ Colic N = 13} & Industry & $10(76.9)$ & $2(15.4)$ & & 0.657 & 1.00 \\
\hline & None/Not clear & & & & & \\
\hline & Non industry & $1(7.7)$ & & & & \\
\hline \multirow[t]{3}{*}{ Spitting up/Regurgitation $N=26$} & Industry & $19(73.1)$ & $1(3.8)$ & & 0.032 & 0.062 \\
\hline & None/Not clear & $2(7.7)$ & $2(7.7)$ & & & \\
\hline & Non industry & $2(7.7)$ & & & & \\
\hline \multirow[t]{3}{*}{ Vomiting $N=32$} & Industry & $19(59.4)$ & $5(15.6)$ & & & \\
\hline & None/Not clear & $3(9.4)$ & $3(9.4)$ & & & \\
\hline & Non industry & $2(6.3)$ & & & & \\
\hline \multirow[t]{3}{*}{ Crying Fussiness $N=20$} & Industry & $10(50)$ & $6(30)$ & & 0.648 & 1.00 \\
\hline & None/Not clear & $2(10)$ & $2(10)$ & & & \\
\hline & Non industry & 0 & & & & \\
\hline \multirow{3}{*}{$\begin{array}{l}\text { Gastric residuals, Abdominal } \\
\text { distension } N=6\end{array}$} & Industry & & $1(16.7)$ & & 0.513 & 1.00 \\
\hline & None/Not clear & $1(16.7)$ & $1(16.7)$ & & & \\
\hline & Non industry & $2(33.3)$ & $1(16.7)$ & & & \\
\hline \multirow{3}{*}{$\begin{array}{l}\text { Volume of formula consumed/daily } \\
\text { intake } N=30\end{array}$} & Industry & $19(63.3)$ & $2(6.7)$ & $1(3.3)$ & 0.867 & 0.733 \\
\hline & None/Not clear & $3(10.0)$ & & & & \\
\hline & Non industry & $4(13.3)$ & $1(3.3)$ & & & \\
\hline \multirow[t]{3}{*}{ Days to full enteral feeding $N=8$} & Industry & $2(25)$ & & $1(12.5)$ & 0.547 & 1.00 \\
\hline & None/Not clear & $1(12.5)$ & $1(12.5)$ & & & \\
\hline & Non industry & 2() 25 & $1(12.5)$ & & & \\
\hline
\end{tabular}

$\$ \$$ Overall percentage. 
Table 15 Association between sponsor and conclusion on reported outcome: Stool characteristics

\begin{tabular}{|c|c|c|c|c|c|c|}
\hline Stool characteristics & Source of funding & $\frac{\text { No conclusion on reported outcome }}{n(\%)^{\$ s}}$ & $\frac{\text { Positive }}{n(\%)^{\$ \$}}$ & $\frac{\text { Negative }}{\mathrm{n}(\%)^{\$ \$}}$ & $\begin{array}{l}\text { Pearson's } \\
\text { chi Square }\end{array}$ & $\begin{array}{l}\text { Fisher's exact } \\
\text { p value }\end{array}$ \\
\hline \multirow[t]{4}{*}{ Stool frequency $N=38$} & Industry & $21(55.3)$ & $9(23.7)$ & & 0.809 & 1.00 \\
\hline & None / Not clear & $5(13.2)$ & $2(5.3)$ & & & \\
\hline & Non industry & $1(2.6)$ & & & & \\
\hline & Total & $27(71.1)$ & $11(28.9)$ & & & \\
\hline \multirow[t]{4}{*}{ Stool consistency $n=39$} & Industry & $18(46.2)$ & $13(33.3)$ & & 0.699 & 1.00 \\
\hline & None / Not clear & $4(10.3)$ & $3(7.7)$ & & & \\
\hline & Non industry & $1(2.6)$ & & & & \\
\hline & Total & $23(59)$ & $16(41)$ & & & \\
\hline \multirow[t]{4}{*}{ Stool pH N =12 } & Industry & $5(41.7)$ & $3(25)$ & & 0.679 & 1.00 \\
\hline & None / Not clear & $2(16.7)$ & $2(16.7)$ & & & \\
\hline & Non industry & & & & & \\
\hline & Total & $7(58.3)$ & $5(41.7)$ & & & \\
\hline \multirow[t]{4}{*}{ Stool short chain fatty acids $N=9$} & Industry & $3(33.3)$ & $3(33.3)$ & & 0.638 & 1.00 \\
\hline & None / Not clear & $1(11.1)$ & $1(11.1)$ & & & \\
\hline & Non industry & $1(11.1)$ & & & & \\
\hline & Total & $5(55.6)$ & $4(44.4)$ & & & \\
\hline \multirow[t]{4}{*}{ Flatulence/Gas N = 15} & Industry & $10(66.7)$ & $4(26.7)$ & & 0.533 & 1.00 \\
\hline & None / Not clear & & & & & \\
\hline & Non industry & $1(6.7)$ & & & & \\
\hline & Total & $11(73.3)$ & $4(26.7)$ & & & \\
\hline \multirow[t]{4}{*}{ Diarrhoea, Diarrhoea episodes N = 18} & Industry & $7(38.9)$ & $5(27.8)$ & $1(5.6)$ & 0.484 & 0.557 \\
\hline & None / Not clear & $2(11.1)$ & & & & \\
\hline & Non industry & $3(16.7)$ & & & & \\
\hline & Total & $12(66.7)$ & $5(27.8)$ & $1(5.6)$ & & \\
\hline \multirow[t]{4}{*}{ Constipation $N=4$} & Industry & $2(50)$ & $1(25)$ & & 0.505 & 1.00 \\
\hline & None / Not clear & & & & & \\
\hline & Non industry & $1(25)$ & & & & \\
\hline & Total & $3(75)$ & $1(25)$ & $1(25)$ & & \\
\hline
\end{tabular}

$\$ \$$ Overall percentage.

biomedical research is increasingly being funded by industry $[1,2]$ There was a trend that more RCTs on preterm infants failed to report their source of funding. The reason(s) for this trend needs to be explored further.

Cochrane guidelines were used to assess the risk of bias of included RCTs. The reporting of several domains was however suboptimal particularly sequence generation, allocation concealment and blinding domains. Considering completed data, there was no significant association between funding source and methodological quality of RCTs in the domains of sequence generation, allocation concealment, blinding and selective reporting. There was a significant association between funding and methodological quality of RCTs in the domains of incomplete outcome data and free of other bias. Industry funded trials had significantly less missing data than non-industry funded trials. A higher percentage of industry funded trials were free of other bias compared to non-industry funded trials. More industry sponsored trials had low risk of bias in 5 out of 6 domains, even though our results did not show a statistical significant association between funding and methodological quality in most domains. Our results confirm findings from previous reviews on infants given enteral feeds with probiotics, prebiotics and synbiotics [157-159].

There was no significant association between funding source and clinical outcomes or majority of authors' conclusions. There was a significant association between funding and conclusion on weight gain. Regardless of the reported clinical outcomes, nearly all RCTs in this review reported neutral results. That is supplementation with probiotics, prebiotics or synbiotics did not have a significant effect or there were no significant differences between study groups of infants given supplemented formula or placebo. Our findings confirm the results of two 
Table 16 Association between sponsor and conclusion on reported outcome: Microflora

\begin{tabular}{|c|c|c|c|c|c|c|c|}
\hline \multirow[t]{2}{*}{ Microflora } & \multirow[t]{2}{*}{ Source of funding } & \multirow{2}{*}{$\frac{\text { No conclusion on reported outcome }}{\mathrm{n}(\%)^{\$ \$}}$} & \multirow{2}{*}{$\frac{\text { Positive }}{\mathrm{n}(\%)^{\$ \$}}$} & \multirow{2}{*}{$\frac{\text { Negative }}{\mathrm{n}(\%)^{\$ \$}}$} & \multirow{2}{*}{$\frac{\text { Neutral }}{n(\%)^{\$ \$}}$} & \multirow{2}{*}{$\begin{array}{l}\text { Chi-square } \\
p \text { value }\end{array}$} & \multirow{2}{*}{$\begin{array}{l}\text { Fisher's exact } \\
\text { p value }\end{array}$} \\
\hline & & & & & & & \\
\hline \multirow[t]{3}{*}{ Bifidobacteria N = 30} & Industry & $7(23.3)$ & $11(36.7)$ & & & 0.249 & 0.195 \\
\hline & None/Not clear & $2(6.7)$ & $5(16.7)$ & $1(3.3)$ & $1(3.3)$ & & \\
\hline & Non industry & $1(3.3)$ & $1(3.3)$ & & $1(3.3)$ & & \\
\hline \multirow[t]{3}{*}{ Lactobacillus N = 19} & Industry & $5(26.3)$ & $4(21.1)$ & & & 0.084 & 0.294 \\
\hline & None/Not clear & $3(15.8)$ & $4(21.1)$ & & $1(5.3)$ & & \\
\hline & Non industry & $1(5.3)$ & & $1(5.3)$ & & & \\
\hline \multirow[t]{3}{*}{ Pathogens N = 25} & Industry & $7(28)$ & $6(24)$ & & & 0.152 & 0.269 \\
\hline & None/Not clear & $4(16)$ & $5(20)$ & $1(4)$ & & & \\
\hline & Non industry & $1(4)$ & & $1(4)$ & & & \\
\hline
\end{tabular}

${ }^{\$}$ Overall percentage.

systematic reviews which found that supplementation with probiotics, prebiotics or synbiotics did not offer any distinct advantage over placebo [158,159]. However, results of this review did not agree with two nutrition related reviews or reviews on pharmaceutical industry supported RCTs, which reported that industry sponsored RCTs had results and conclusions in favour of the sponsor [2-4,6,8,160-162]. Despite reporting neutral outcomes, authors from industry sponsored RCTs had a tendency to advocate for the consumption of the sponsors' products. Similar findings were reported by Nestle, who reported that research investigators "who received company grants tended to publish results, give advice and prescribe in favour of the sponsor." This applied to research that was supported by pharmaceutical and food industries [163].

Effects of sponsorship on overall study conclusion have been equally documented in biomedical literature. Reviews by Lessor and Nkansah reported positive conclusions in favour of the sponsor [6,8]. Although no statistically significant association between funding and authors conclusion was found in this review, more than $70 \%$ of RCTs reported positive conclusions, $47.8 \%$ of these were industry sponsored. Often, these positive conclusions in the RCTs were not supported by the reported data as demonstrated by the neutral clinical outcomes. Our findings are consistent with those of previous reviews, which found that, results from RCTs may be accurate, but authors may distort the meaning of the results, present conclusions that are more favourable, and that were not supported by the data presented $[2,5,163]$. Even meta - analyses were not spared from this trend $[2,5,163]$. Despite overwhelming positive overall study conclusions, majority of RCTs did not have any conclusion on their reported clinical outcomes. The RCTs that reported any conclusion on their clinical outcomes, majority were positive in favour of the sponsors' products.

\section{Limitations}

This review did not document the role of the sponsor in study design, data collection, and analysis. Few RCTs

Table 17 Association between sponsor and conclusion on reported outcome: Necrotising Enterocolitis Sepsis and antibiotic use

\begin{tabular}{|c|c|c|c|c|c|c|}
\hline & Source of funding & $\frac{\text { No conclusion on reported outcome }}{\mathrm{n}(\%)^{\$ \$}}$ & $\frac{\text { Positive }}{n(\%)^{\$ \$}}$ & $\frac{\text { Negative }}{\mathrm{n}(\%)^{\$ \$}}$ & $\begin{array}{l}\text { Pearson's } \\
\text { chi Square }\end{array}$ & $\begin{array}{l}\text { Fisher's exact } \\
\text { p value }\end{array}$ \\
\hline \multirow[t]{4}{*}{$\mathrm{NEC} \mathrm{N}=12$} & Industry & $3(25)$ & & $1(8.3)$ & 0.511 & 0.782 \\
\hline & None/Not clear & $2(16.7)$ & $1(8.3)$ & $1(8.3)$ & & \\
\hline & Non industry & $2(16.7)$ & $2(16.7)$ & 0 & & \\
\hline & & $7(58.3)$ & $3(25)$ & $2(16.7)$ & & \\
\hline \multirow[t]{3}{*}{ Sepsis N = 10} & Industry & $2(20)$ & & & 0.274 & 0.500 \\
\hline & None/Not clear & $2(20)$ & & $1(10)$ & & \\
\hline & Non industry & $5(50)$ & & & & \\
\hline \multirow[t]{3}{*}{ Antibiotic use $\mathrm{N}=16$} & Industry & $4(25)$ & $3(18.8)$ & & 0.093 & 0.141 \\
\hline & None/Not clear & $4(25)$ & & & & \\
\hline & Non industry & $5(31.3)$ & & & & \\
\hline
\end{tabular}

\$\$ Overall percentage. 
reported this. More detailed documentation and disclosure in RCT reports would help evaluate if there was an association between funding and reported outcomes or conclusions. Many RCTs had missing data especially on the domains of sequence generation, allocation concealment and blinding. Attempts were made to contact authors for missing information but none responded. The sample size (number of RCTs) was small and skewed towards industry.

\section{Conclusion}

This study assessed the impact of funding by the food industry on trial outcomes and methodological quality of synbiotics, probiotics and prebiotics research in infants. There was no significant association between source of funding and methodological quality of study in the domains of sequence generation, allocation concealment and blinding. Industry funded trials had less missing data and were free of other bias than non-industry funded trials.

There was no significant association between funding and majority of reported clinical outcomes or authors' conclusions. However, there was a significant association between funding source and reported antibiotic use and conclusion on weight gain. Majority of RCTs were industry funded, more non-industry funded research is needed to further assess the impact of funding on methodological quality, reported clinical outcomes and authors' conclusions.

\section{Competing interests}

The authors declare that they have no competing interests.

\section{Authors' contributions}

The reviewers contributed the following: MM: Developed review protocol (unpublished), selected RCTs, conducted data extraction, assessment of risk of bias in included RCTs, developed, edited and critically reviewed the manuscript. ML: Selected RCTs, conducted data extraction, assessment of risk of bias in included RCTs, critically reviewed the manuscript. AM: Conducted the statistical analysis, interpretation of results and critically reviewed the manuscript. TY: Contributed to designing the review methodology and critically reviewed the manuscript. RB: Contributed to designing the review, acted as third party arbitrator and critically reviewed the manuscript. All authors read and approved the final manuscript.

\section{Acknowledgements \\ This review was supported through a grant from Stellenbosch University, Faculty of Medicine and Health Sciences. South Africa. The sponsors had no role in study design, data collection, analysis and interpretation, report writing or conclusions reached in this review.}

\section{Author details}

'Division of Human Nutrition, Faculty of Medicine and Health Sciences, Stellenbosch University, P.O Box 19063, Tygerberg 7505, South Africa. ${ }^{2}$ Centre for Evidence-Based Health Care, Faculty of Medicine and Health Sciences, Stellenbosch University, Stellenbosch, South Africa.

Received: 19 July 2013 Accepted: 7 November 2013

Published: 13 November 2013

\section{References}

1. Khan NA, Lombeida Jl, Singh M, Spencer HJ, Torralba KD: Association of industry funding with the outcome and quality of randomized controlled trials of drug therapy for rheumatoid arthritis. Arthritis Rheum 2012, 64:2059-2067.

2. Lexchin J: Those who have the gold make the evidence: How the pharmaceutical industry biases the outcomes of clinical trials of medications. Sci Eng Ethics 2012, 18:247-261.

3. Sismondo S: Pharmaceutical company funding and its consequences: A qualitative systematic review. Contemp Clin Trials 2008, 29:109-113.

4. Bekelman JE, Li Y, Gross CP: Scope and impact of financial conflicts of interest in biomedical research: A systematic review. JAMA 2003, 289:454-466.

5. Als-Nielsen B, Chen W, Gluud C, Kjaergard LL: Association of funding and conclusions in randomized drug trials: A reflection of treatment effect or adverse events? J Am Med Assoc 2003, 290:921-928.

6. Lesser LI, Ebbeling CB, Goozner M, Wypij D, Ludwig DS: Relationship between funding source and conclusion among nutrition-related scientific articles. PLoS Med 2007, 4:0041-0046.

7. Katan MB: Does industry sponsorship undermine the integrity of nutrition research? PLoS Med 2007, 4:0003-0004.

8. Nkansah N, Nguyen T, Iraninezhad H, Bero L: Randomized trials assessing calcium supplementation in healthy children: Relationship between industry sponsorship and study outcomes. Public Health Nutr 2009, 12:1931-1937.

9. Shah NP: Functional cultures and health benefits. Int Dairy J 2007, 17:1262-1277

10. Gibson GR, Delzenne N: Inulin and oligofructose: New scientific developments. Nutr Today 2008, 43:54-59.

11. Gibson GR: Fibre and effects on probiotics (the prebiotic concept). Clin Nutr Supp/ 2004, 1:25-31.

12. Tuohy KM, Probert HM, Smejkal CW, Gibson GR: Using probiotics and prebiotics to improve health. DDT 2003, 8:692-700.

13. Macfarlane GT, Steed H, Macfarlane S: Bacterial metabolism and health-related effects of galactooligosaccharides and other prebiotics. J Appl Microbiol 2008, 104:305-344.

14. Losada MA, Olleros T: Towards a healthier diet for the colon: The influence of fructooligosaccharides and lactobacilli on intestinal health. Nutr Res 2002, 22:71-84.

15. Watzl B, Girrbach S, Roller M: Inulin, oligofructose and immunomodulation. Br J Nutr 2005, 93(Suppl S1):S49-S55.

16. Torres DP, Gonçalves M, Teixeira JA, Rodrigues LR: Galactooligosaccharides: Production, properties, applications, and significance as prebiotics. Compr Rev Food Sci Food Saf. 2010, 9:438-454.

17. Higgins JPT, Green S: Cochrane handbook for systematic reviews of interventions. John Wiley \& Sons Ltd: Chichester, West Sussex; 2008.

18. Allen SJ, Jordan S, Storey M, Thornton CA, Gravenor M, Garaiova I, Plummer SF, Wang D, Morgan G: Dietary supplementation with lactobacilli and bifidobacteria is well tolerated and not associated with adverse events during late pregnancy and early infancy. J Nutr 2010, 140:483-488.

19. Soh SE, Aw M, Gerez I, Chong YS, Rauff M, Ng YPM, Wong HB, Pai N, Lee BW, Shek LP: Probiotic supplementation in the first 6 months of life in at risk asian infants - effects on eczema and atopic sensitization at the age of 1 year. Clin Exp Allergy 2009, 39:571-578.

20. Jacobs S: The use of probiotics to reduce the incidence of sepsis in premature infants. : Australian New Zealand Clinical Trials Registry; 2007. ACTRN126070001444415 26/02/2007 [http://www.anzctr.org.au].

21. Alliet $P$, Scholtens $P$, Raes $M$, Hensen $K$, Jongen $H$, Rummens J, Boehm $G$, Vandenplas $Y$ : Effect of prebiotic galacto-oligosaccharide, long-chain fructo-oligosaccharide infant formula on serum cholesterol and triacylglycerol levels. Nutrition 2007, 23:719-723.

22. Scholtens PAMJ, Alliet P, Raes M, Alles MS, Kroes H, Boehm G, Knippels LMJ, Knol J, Vandenplas $Y$ : Fecal secretory immunoglobulin $A$ is increased in healthy infants who receive a formula with short-chain galacto-oligosaccharides and long-chain fructo-oligosaccharides. J Nutr 2008, 138:1141-1147.

23. Urban MF, Bolton KD, Mokhachane M, Mphahlele RM, Bomela HN, Monaheng L, Beckh-Arnold E, Cooper PA: Growth of infants born to HIV-infected women when fed a biologically acidified starter formula with and without probiotics. South Afr J Clin Nutr 2008, 21:28-32.

24. Patole S: A randomized placebo controlled trial on the safety and efficacy of a probiotic product in reducing all case mortality and definite necrotising enterocolitis in preterm very low birth weight neonates. 
In Australian New Zealand Clinical Trials Registry. 2009. ACTRN12609000374268 27/05/2009 [http://www.anzctr.org.au].

25. Ashley C, Johnston WH, Harris CL, Stolz SI, Wampler JL, Berseth CL: Growth and tolerance of infants fed formula supplemented with polydextrose (PDX) and/or galactooligosaccharides (GOS): Double-blind, randomized, controlled trial. Nutr J 2012, 11:38. doi:10.1186/1475-2891-11-38.

26. Velaphi SC, Cooper PA, Bolton KD, Mokhachane M, Mphahlele RM, BeckhArnold E, Monaheng L, Haschke-Becher E: Growth and metabolism of infants born to women infected with human immunodeficiency virus and fed acidified whey-adapted starter formulas. Nutrition 2008, 24:203-211.

27. Underwood M: The impact of oligosaccharides and bifidobacteria on the intestinal microflora of Premature infants. Clinical trials registry. ; NCT00810160 05/11/2009 [http://www.clinicaltrials.gov].

28. Bakker-Zierikzee AM, Alles MS, Knol J, Kok FJ, Tolboom JJM, Bindels JG: Effects of infant formula containing a mixture of galacto- and fructo-oligosaccharides or viable bifidobacterium animalis on the intestinal microflora during the first 4 months of life. Br J Nutr 2005, 94:783-790

29. Bakker-Zierikzee AM, Van Tol EAF, Kroes H, Alles MS, Kok FJ, Bindels JG: Faecal SlgA secretion in infants fed on pre- or probiotic infant formula. Pediatr Allergy Immunol 2006, 17:134-140.

30. Vendt N, Grünberg H, Tuure T, Malminiemi O, Wuolijoki E, Tillmann V, Sepp E, Korpela R: Growth during the first 6 months of life in infants using formula enriched with lactobacillus rhamnosus GG: Double-blind, randomized trial. J Hum Nutr Diet 2006, 19:51-58.

31. Bettler J, Euler AR: An evaluation of the growth of term infants fed formula supplemented with fructo-oligosaccharide. Int J Probiotics Prebiotics 2006, 1:19-26.

32. Vieger AM, Robroch A, Van Buuren S, Kiers J, Rijkers G, Benninga MA, Te Biesebeke $R$ : Tolerance and safety of lactobacillus paracasei ssp. paracasei in combination with bifidobacterium animalis ssp. lactis in a prebiotic-containing infant formula: A randomised controlled trial. Br J Nutr 2009, 102:869-875.

33. Brunser O, Figueroa G, Gotteland M, Haschke-Becher E, Magliola C, Rochat F, Cruchet S, Palframan R, Gibson G, Chauffard F, Haschke F: Effects of probiotic or prebiotic supplemented milk formulas on fecal microbiota composition of infants. Asia Pac J Clin Nutr 2006, 15:368-376.

34. Weizman Z, Asli G, Alsheikh A: Effect of a probiotic infant formula on infections in child care centers: Comparison of two probiotic agents. Pediatrics 2005, 115:5-9.

35. Bruzzese $E$, Volpicelli M, Squeglia V, Bruzzese D, Salvini F, Bisceglia M, Lionetti $P$, Cinquetti M, lacono G, Amarri S, Guarino A: A formula containing galacto- and fructo-oligosaccharides prevents intestinal and extra-intestinal infections: An observational study. Clin Nutr 2009, 28:156-161.

36. Weizman Z, Alsheikh A: Safety and tolerance of a probiotic formula in early infancy comparing two probiotic agents: A pilot study. J Am Coll Nutr 2006, 25:415-419.

37. Chouraqui JP, Van Egroo LD, Fichot MC: Acidified milk formula supplemented with bifidobacterium lactis: Impact on infant diarrhea in residential care settings. J Pediatr Gastroenterol Nutr 2004, 38:288-292.

38. Xiao-Ming B, Zhou X-Y, Zhao W-H, Yu W-L, Pan W, Zhang W-L, Wu S-M, Van Beusekom CM, Schaafsma A: Supplementation of milk formula with galacto-oligosaccharides improves intestinal micro-flora and fermentation in term infants. Chin Med J 2004, 117:927-931.

39. Chouraqui JP, Grathwohl D, Labaune JM, Hascoet JM, De Montgolfier I, Leclaire M, Giarre M, Steenhout P: Assessment of the safety, tolerance, and protective effect against diarrhea of infant formulas containing mixtures of probiotics or probiotics and prebiotics in a randomized controlled trial. Am J Clin Nutr 2008, 87:1365-1373.

40. Xiao-Ming B, Li J, Feng Z-T, Shi S-Y, Lu Y-D, Chen R, Zhou X-Y: Low level of galacto-oligosaccharide in infant formula stimulates growth of intestinal bifidobacteria and lactobacilli. World J Gastroenterol 2008, 14:6564-6568.

41. Cooper PA, Bolton KD, Mokhachane M, Velaphi SC, Mphahlele RM, Bomela $H N$, Monaheng L, Roux P, Haschke-Becher E: Growth of infants born to HIV-positive mothers fed a whey-adapted acidifed starter formula with prebiotics and nucleotides. South Afr J Clin Nutr 2010, 23:90-95.

42. Ziegler E, Vanderhoof JA, Petschow B, Mitmesser SH, Stolz SI, Harris CL, Berseth $\mathrm{CL}$ : Term infants fed formula supplemented with selected blends of prebiotics grow normally and have soft stools similar to those reported for breast-fed infants. J Pediatr Gastroenterol Nutr 2007, 44:359-364.
43. Costalos C, Kapiki A, Apostolou M, Papathoma E: The effect of a prebiotic supplemented formula on growth and stool microbiology of term infants. Early Hum Dev 2008, 84:45-49.

44. Bin-Nun A, Bromiker R, Wilschanski M, Kaplan M, Rudensky B, Caplan M, Hammerman C: Oral probiotics prevent necrotizing enterocolitis in very low birth weight neonates. J Pediatr 2005, 147:192-196.

45. Decsi T, Arató A, Balogh M, Dolinay T, Kanjo AH, Szabó É, Várkonyi Á: Randomised placebo controlled double blind study on the effect of prebiotic oligosaccharides on intestinal flora in healthy infants. Orv Hetil 2005, 146:2445-2450.

46. Boehm G, Lidestri M, Casetta P, Jelinek J, Negretti F, Stahl B, Marini A: Supplementation of a bovine milk formula with an oligosaccharide mixture increases counts of faecal bifidobacteria in preterm infants. Arch Dis Child Fetal Neonatal Ed 2002, 86:F178-F181

47. Boehm G, Fanaro S, Jelinek J, Stahl B, Marini A: Prebiotic concept for infant nutrition. Acta Paediatr 2003, 91:64-67.

48. Knol J, Boehm G, Lidestri M, Negretti F, Jelinek J, Agosti M, Stahl B, Marini A, Mosca F: Increase of faecal bifidobacteria due to dietary oligosaccharides induces a reduction of clinically relevant pathogen germs in the faeces of formula-fed preterm infants. Acta Paediatr 2005, 94:31-33.

49. Fanaro S, Jelinek J, Stahl B, Boehm G, Kock R, Vigi V: Acidic oligosaccharides from pectin hydrolysate as new component for infant formulae: Effect on intestinal flora, stool characteristics, and $\mathrm{pH}$. J Pediatr Gastroenterol Nutr 2005, 41:186-190.

50. Chrzanowska-Liszewska D, Seliga-Siwecka J, Kornacka MK: The effect of lactobacillus rhamnosus GG supplemented enteral feeding on the microbiotic flora of preterm infants-double blinded randomized control trial. Early Hum Dev 2012, 88:57-60.

51. Fanaro S, Marten B, Bagna R, Vigi V, Fabris C, Peña-Quintana L, Argüelles F, Scholz-Ahrens KE, Sawatzki G, Zelenka R, Schrezenmeir J, De Vrese M, Bertino E: Galacto-oligosaccharides are bifidogenic and safe at weaning: A double-blind randomized multicenter study. J Pediatr Gastroenterol Nutr 2009, 48:82-88.

52. Costalos C, Skouteri V, Gounaris A, Sevastiadou S, Triandafilidou A, Ekonomidou C, Kontaxaki F, Petrochilou V: Enteral feeding of premature infants with saccharomyces boulardii. Early Hum Dev 2003, 74:89-96.

53. Gibson RA, Barclay D, Marshall H, Moulin J, Maire J, Makrides M: Safety of supplementing infant formula with long-chain polyunsaturated fatty acids and bifidobacterium lactis in term infants: A randomised controlled trial. Br J Nutr 2009, 101:1706-1713.

54. Dani C, Biadaioli R, Bertini G, Martelli E, Rubaltelli FF: Probiotics feeding in prevention of urinary tract infection, bacterial sepsis and necrotizing enterocolitis in preterm infants: A prospective double-blind study. Biol Neonate 2002, 82:103-108.

55. Gil-Campos M, López MÁ, Rodriguez-Benítez MV, Romero J, Roncero I, Linares MD, Maldonado J, López-Huertas E, Berwind R, Ritzenthaler KL, Navas V, Sierra C, Sempere L, Geerlings A, Maldonado-Lobón JA, Valero AD, LaraVilloslada F, Olivares M: Lactobacillus fermentum CECT 5716 is safe and well tolerated in infants of 1-6 months of age: A randomized controlled trial. Pharmacol Res 2012, 65:231-238.

56. Indrio F, Riezzo G, Raimondi F, Bisceglia M, Cavallo L, Francavilla R: The effects of probiotics on feeding tolerance, bowel habits, and gastrointestinal motility in preterm newborns. J Pediatr 2008, 152:801-806.

57. Hascoët J, Hubert C, Rochat F, Legagneur H, Gaga S, Emady-Azar S, Steenhout PG: Effect of formula composition on the development of infant gut microbiota. J Pediatr Gastroenterol Nutr 2011, 52:756-762.

58. Indrio F, Riezzo G, Raimondi F, Francavilla R, Montagna O, Valenzano ML, Cavallo L, Boehm G: Prebiotics improve gastric motility and gastric electrical activity in preterm newborns. J Pediatr Gastroenterol Nutr 2009, 49:258-261.

59. Holscher HD, Czerkies LA, Cekola P, Litov R, Benbow M, Santema S, Alexander DD, Perez V, Sun S, Saavedra JM, Tappenden KA: Bifidobacterium lactis Bb12 enhances intestinal antibody response in formula-fed infants: A randomized, double-blind, controlled trial. J Parenter Enteral Nutr 2012, 36(Suppl 1):106S-117S.

60. Kapiki A, Costalos C, Oikonomidou C, Triantafyllidou A, Loukatou E, Pertrohilou $\checkmark$ : The effect of a fructo-oligosaccharide supplemented formula on gut flora of preterm infants. Early Hum Dev 2007, 83:335-339.

61. Holscher HD, Faust KL, Czerkies LA, Litov R, Ziegler EE, Lessin H, Hatch T, Sun S, Tappenden KA: Effects of prebiotic-containing infant formula on gastrointestinal tolerance and fecal microbiota in a randomized controlled trial. J Parenter Enteral Nutr 2012, 36(Suppl 1):95S-105S. 
62. Kitajima H, Sumida Y, Tanaka R, Yuki N, Takayama H, Fujimura M: Early administration of bifidobacterium breve to preterm infants: Randomised controlled trial. Arch Dis Child Fetal Neonatal Ed 1997, 76:F101-F107.

63. Kim JY, Kwon JH, Ahn SH, Lee SI, Han YS, Choi YO, Lee SY, Ahn KM, Ji GE: Effect of probiotic mix (bifidobacterium bifidum, bifidobacterium lactis, lactobacillus acidophilus) in the primary prevention of eczema: A double-blind, randomized, placebo-controlled trial. Pediatr Allergy Immunol 2010, 21:e386-e393.

64. Lin H, Hsu C, Chen H, Chung M, Hsu J, Lien R, Tsao L, Chen C, B- S: Oral probiotics prevent necrotizing enterocolitis in very low birth weight preterm infants: A multicenter, randomized, controlled trial. Pediatrics 2008, 122:693-700.

65. Knol J, Scholtens P, Kafka C, Steenbakkers J, Groß S, Helm K, Klarczyk M, Schöpfer $\mathrm{H}$, Böckler $\mathrm{H}$, Wells J: Colon microflora in infants fed formula with galacto- and fructo-oligosaccharides: More like breast-fed infants. J Pediatr Gastroenterol Nutr 2005, 40:36-42.

66. Mihatsch W, Hoegel J, Pohlandt F: Prebiotic oligosaccharides reduce stool viscosity and accelerate gastrointestinal transport in preterm infants. Acta Paediatr 2006, 95:843-848.

67. Magne F, Hachelaf W, Suau A, Boudraa G, Bouziane-Nedjadi K, Rigottier-Gois $L$, Touhami M, Desjeux J, Pochart P: Effects on faecal microbiota of dietary and acidic oligosaccharides in children during partial formula feeding. J Pediatr Gastroenterol Nutr 2008, 46:580-588.

68. Mihatsch WA, Vossbeck S, Eikmanns B, Hoegel J, Pohlandt F: Effect of bifidobacterium lactis on the incidence of nosocomial infections in verylow-birth-weight infants: A randomized controlled trial. Neonatology 2010, 98:156-163.

69. Mah KW, Chin VIL, Wong WS, Lay C, Tannock GW, Shek LP, Aw MM, Chua $\mathrm{KY}$, Wong HB, Panchalingham A, Lee BW: Effect of a milk formula containing probiotics on the fecal microbiota of asian infants at risk of atopic diseases. Pediatr Res 2007, 62:674-679.

70. Millar MR, Bacon C, Smith SL, Walker V, Hall MA: Enteral feeding of premature infants with lactobacillus GG. Arch Dis Child 1993, 69(Suppl 5):483-487.

71. Stansbridge EM, Walker V, Hall MA, Smith SL, Millar MR, Bacon C, Chen S: Effects of feeding premature infants with lactobacillus GG on gut fermentation. Arch Dis Child 1993, 69(Suppl 5):488-492.

72. Maldonado J, Lara-Villoslada F, Sierra S, Sempere L, Gómez M, Rodriguez JM, Boza J, Xaus J, Olivares M: Safety and tolerance of the human milk probiotic strain lactobacillus salivarius CECT5713 in 6-month-old children. Nutrition 2010, 26:1082-1087.

73. Modi N, Uthaya S, Fell J, Kulinskaya E: A randomized, double-blind, controlled trial of the effect of prebiotic oligosaccharides on enteral tolerance in preterm infants (ISRCTN77444690). Pediatr Res 2010, 68:440-445.

74. Moro G, Minoli I, Mosca M, Fanaro S, Jelinek J, Stahl B, Boehm G: Dosage-related bifidogenic effects of galacto- and fructooligosaccharides in formula-fed term infants. J Pediatr Gastroenterol Nutr 2002, 34:291-295.

75. Moro GE, Mosca F, Miniello V, Fanaro S, Jelinek J, Stahl B, Boehm G: Effects of a new mixture of prebiotics on faecal flora and stools in term infants. Acta Paediatr 2003, 91(Suppl 441):77-79.

76. Mohan R, Koebnick C, Schildt J, Schmidt S, Mueller M, Possner M, Radke M, Blaut M: Effects of bifidobacterium lactis Bb12 supplementation on intestinal microbiota of preterm infants: A double-blind, placebocontrolled, randomized study. J Clin Microbiol 2006, 44:4025-4031.

77. Moro GE, Stahl B, Fanaro S, Jelinek J, Boehm G, Coppa GV: Dietary prebiotic oligosaccharides are detectable in the faeces of formula-fed infants. Acta Paediatr 2005, 94(Supple 449):27-30

78. Reuman PD, Duckworth DH, Smith KL, Kagan R, Bucciarelli RL, Ayoub EM: Lack of effect of lactobacillus on gastrointestinal bacterial colonization in premature infants. Pediatr Infect Dis 1986, 5:663-668.

79. Moro G, Arslanoglu S, Stahl B, Jelinek J, Wahn U, Boehm G: A mixture of prebiotic oligosaccharides reduces the incidence of atopic dermatitis during the first six months of age. Arch Dis Child 2006, 91:814-819.

80. Arslanoglu S, Moro GE, Boehm G: Early supplementation of prebiotic oligosaccharides protects formula-fed infants against infections during the first 6 months of life. J Nutr 2007, 137:2420-2424.

81. Arslanoglu S, Moro GE, Schmitt J, Tandoi L, Rizzardi S, Boehm G: Early dietary intervention with a mixture of prebiotic oligosaccharides reduces the incidence of allergic manifestations and infections during the first two years of life. J Nutr 2008, 138:1091-1095.

82. Van Hoffen E, Ruiter B, Faber J, M'Rabet L, Knol EF, Stahl B, Arslanoglu S, Moro G, Boehm G, Garssen J: A specific mixture of short-chain galacto-oligosaccharides and long-chain fructo-oligosaccharides induces a beneficial immunoglobulin profile in infants at high risk for allergy. Allergy 2009, 64:484-487.

83. Schouten B, van Esch BCAM, Kormelink TG, Moro GE, Arslanoglu S, Boehm G, Knippels LMJ, Redegeld FA, Willemsen LEM, Garssen J: Non-digestible oligosaccharides reduce immunoglobulin free light-chain concentrations in infants at risk for allergy. Pediatr Allergy Immunol 2011, 22:537-542.

84. Riskin A, Hochwald O, Bader D, Srugo I, Naftali G, Kugelman A, Cohen E, Mor F, Kaufman B, Shaoul R: The effects of lactulose supplementation to enteral feedings in premature infants: A pilot study. J Pediatr 2010, 156:209-214.

85. Piemontese $P$, Giannì $M L$, Braegger $C P$, Chirico $G$, Grüber $C$, Riedler J, Arslanoglu S, van Stuijvenberg M, Boehm G, Jelinek J, Roggero P: Tolerance and safety evaluation in a large cohort of healthy infants fed an innovative prebiotic formula: A randomized controlled trial. PLOS One 2011, 6(11):e28010. doi:10.1371/journal.pone.0028010.

86. Rougé C, Piloquet $H$, Butel M, Berger B, Rochat F, Ferraris L, Des Robert C, Legrand A, De La Cochetière M, N'Guyen J, Vodovar M, Voyer M, Darmaun D, Rozé J: Oral supplementation with probiotics in very-low-birth-weight preterm infants: A randomized, double-blind, placebo-controlled trial. Am J Clin Nutr 2009, 89:1828-1835.

87. Puccio G, Cajozzo C, Meli F, Rochat F, Grathwohl D, Steenhout P: Clinical evaluation of a new starter formula for infants containing live bifidobacterium longum BL999 and prebiotics. Nutrition 2007, 23:1-8.

88. Sari FN, Dizdar EA, Oguz S, Erdeve O, Uras N, Dilmen U: Oral probiotics: Lactobacillus sporogenes for prevention of necrotizing enterocolitis in very low-birth weight infants: A randomized, controlled trial. Eur J Clin Nutr 2011, 65:434-439.

89. Rautava S, Arvilommi H, Isolauri E: Specific probiotics in enhancing maturation of $\lg A$ responses in formula-fed infants. Pediatr Res 2006, 60:221-224.

90. Rautava S, Salminen S, Isolauri E: Specific probiotics in reducing the risk of acute infections in infancy - A randomised, double-blind, placebocontrolled study. Br J Nutr 2009, 101:1722-1726.

91. Stratiki Z, Costalos C, Sevastiadou S, Kastanidou O, Skouroliakou M, Giakoumatou A, Petrohilou V: The effect of a bifidobacter supplemented bovine milk on intestinal permeability of preterm infants. Early Hum Dev 2007, 83:575-579.

92. Rinne MM, Gueimonde M, Kalliomäki M, Hoppu U, Salminen SJ, Isolauri E: Similar bifidogenic effects of prebiotic-supplemented partially hydrolyzed infant formula and breastfeeding on infant gut microbiota. FEMS Immunol Med Microbiol 2005, 43:59-65.

93. Westerbeek EAM, Van Den Berg JP, Lafeber HN, Fetter WPF, Boehm G, Twisk JWR, Van Elburg RM: Neutral and acidic oligosaccharides in preterm infants: A randomized, double-blind, placebo-controlled trial. Am J Clin Nutr 2010, 91:679-686.

94. Westerbeek EAM, Hensgens RL, Mihatsch WA, Boehm G, Lafeber HN, Van Elburg RM: The effect of neutral and acidic oligosaccharides on stool viscosity, stool frequency and stool $\mathrm{pH}$ in preterm infants. Acta Paediatr 2011, 100:1426-1431.

95. Westerbeek EAM, Van Den Berg A, Lafeber HN, Fetter WPF, Van Elburg RM: The effect of enteral supplementation of a prebiotic mixture of nonhuman milk galacto-, fructo- and acidic oligosaccharides on intestinal permeability in preterm infants. Br J Nutr 2011, 105:268-274.

96. Saavedra JM, Abi-Hanna A, Moore N, Yolken RH: Long-term consumption of infant formulas containing live probiotic bacteria: Tolerance and safety. Am J Clin Nutr 2004, 79:261-267.

97. Yong G, Fang $H$, Shuang-Gen M, Guo-Cheng X: Effect of bifid triple viable on feeding intolerance in preterm infants with very low birth weight. [Clinical study on the effect of probiotic preparation on feeding intolerance in preterm infants with very low birth weight. (from Chinese translation)]. Chinese Journal of Microecology 2009, 21:451-452.

98. Scalabrin DM, Johnston WH, Hoffman DR, P'Pool VL, Harris CL, Mitmesser $\mathrm{SH}$ : Growth and tolerance of healthy term infants receiving hydrolyzed infant formulas supplemented with lactobacillus rhamnosus GG: Randomized, double-blind, controlled trial. Clin Pediatr 2009, 48:734-744.

99. Scalabrin DMF, Mitmesser SH, Welling GW, Harris CL, Marunycz JD, Walker DC, Bos NA, Tölkkö S, Salminen S, Vanderhoof JA: New prebiotic blend of polydextrose and galacto-oligosaccharides has a bifidogenic effect in young infants. J Pediatr Gastroenterol Nutr 2012, 54:343-352.

100. Schmelzle H, Wirth S, Skopnik H, Radke M, Knol J, Böckler HM, Brönstrup A, Wells J, Fusch C: Randomized double blind study of the nutritional 
efficacy and bifidogenicity of a new infant formula containing partially hydrolyzed protein, a high beta-palmitic acid level and nod digestible oligosaccharides. J Pediatr Gastroenterol Nutr 2003, 36:342-351.

101. Agarwal R, Sharma N, Chaudhry R, Deorari A, Paul VK, Gewolb IH, Panigrahi $P$ : Effects of oral lactobacillus $G G$ on enteric microflora in low-birthweight neonates. J Pediatr Gastroenterol Nutr 2003, 36:397-402.

102. Al-Hosni M, Duenas M, Hawk M, Stewart LA, Borghese RA, Cahoon M, Atwood L, Howard D, Ferrelli K, Soll R: Probiotics-supplemented feeding in extremely low-birth-weight infants. J Perinatol 2012, 32:253-259.

103. Fengjuan Z, Zheng-yun S, Beilei C: Oral probiotics and immune function and digestive tract of premature children. (Chinese). J Shandong Univ Health Sci 2008, 46(11):1-6.

104. Morisset M, Aubert-Jacquin C, Soulaines P, Moneret-Vautrin D, Dupont C: A non-hydrolyzed, fermented milk formula reduces digestive and respiratory events in infants at high risk of allergy. Eur J Clin Nutr 2011, 65:175-183.

105. Huet F, Lachambre E, Beck L, Van Egroo L, Sznajder M: Evaluation of a formula with low protein content and supplemented with probiotic agents after breast milk weaning. Arch Pediatr 2006, 13:1309-1315.

106. Agustina R, Lukito W, Firmansyah A, Suhardjo HN, Murniati D, Bindels J: The effect of early nutritional supplementation with a mixture of probiotic, prebiotic, fiber and micronutrients in infants with acute diarrhea in Indonesia. Asia Pac J Clin Nutr 2007, 16:435-442.

107. Akiyama K, Hosono S, Takahashi E, Ishizeki S, Takigawa I, Imura S, Yamauchi K, Yaeshima T, Hayasawa H, Shimamura S: Effects of oral administration of bifidobacterium breve on development of intestinal microflora in extremely premature infants. Acta Neonatol Jpn 1994, 30:130-137.

108. Grześkowiak Ł, Grönlund M, Beckmann C, Salminen S, von Berg A, Isolauri E: The impact of perinatal probiotic intervention on gut microbiota: Double-blind placebo-controlled trials in Finland and Germany. Anaerobe 2012, 18:7-13

109. Andrews BF, Cook LN: Low birth-weight infants fed a new carbohydrate-free formula with different sugars, I. Growth and Clinical Course. Am J Clin Nutr 1969, 22:845-850.

110. Baldeón ME, Naranjo G, Granja D: Effect of infant formula with probiotics on intestinal microbiota. Arch Latinoam Nutr 2008, 58:5-11.

111. Campeotto F, Suau A, Kapel N, Magne F, Viallon V, Ferraris L, Waligora-Dupriet A, Soulaines P, Leroux B, Kalach N, Dupont C, Butel M: A fermented formula in pre-term infants: Clinical tolerance, gut microbiota, down-regulation of faecal calprotectin and up-regulation of faecal secretory lgA. Br J Nutr 2011, 105:1843-1851.

112. Kuitunen M, Kukkonen K, Savilahti E: Pro- and prebiotic supplementation induces a transient reduction in hemoglobin concentration in infants. J Pediatr Gastroenterol Nutr 2009, 49:626-630.

113. Patole SK, Muller R: Does carboxymethylcellulose have a role in reducing time to full enteral feeds in preterm neonates? Int I Clin Pract 2005, 59:544-548

114. Bongers MEJ, De Lorijn F, Reitsma JB, Groeneweg M, Taminiau JAJM, Benninga MA: The clinical effect of a new infant formula in term infants with constipation: A double-blind, randomized cross-over trial. Nutr J 2007, 6:8. doi:10.1186/1475-2891-6-8.

115. Corrêa NBO, Péret Filho LA, Penna FJ, Lima FMLS, Nicoli JR: A randomized formula controlled trial of bifidobacterium lactis and streptococcus thermophilus for prevention of antibiotic-associated diarrhea in infants. J Clin Gastroenterol 2005, 39:385-389.

116. Akiyama K, Shimada M, Ishizeki S, Takigawa I, Imura S, Yamauchi K, Hatano M, Abe N, Yaeshima T, Hayasawa H, Shimamura S: Effects of administration of bifidobacterium in extremely premature infants. Development of intestina microflora by orally administered bifidobacterium longum (in comparison with bifidobacterium breve). Acta Neonatol Jpn 1994b, 30:257-263.

117. Robinson EL, Thompson WL: Effect on weight gain of the addition of lactobacillus acidophilus to the formula of newborn infants. J Pediatr 1952, 41:395-398.

118. Braga TD, Da Silva GAP, De Lira PIC, De Carvalho LM: Efficacy of bifidobacterium breve and lactobacillus casei oral supplementation on necrotizing enterocolitis in very-low-birth-weight preterm infants: A double-blind, randomized, controlled trial. Am J Clin Nutr 2011, 93:81-86.

119. Cukrowska B, Lodínová-Žádníková R, Enders C, Sonnenborn U, Schulze J, Tlaskalová-Hogenová H: Specific proliferative and antibody responses of premature infants to intestinal colonization with nonpathogenic probiotic E. coli strain nissle 1917. Scand J Immunol 2002, 55:204-209.
120. Kukkonen K, Savilahti E, Haahtela T, Juntunen-Backman K, Korpela R, Poussa $\mathrm{T}$, Tuure T, Kuitunen M: Probiotics and prebiotic galacto-oligosaccharides in the prevention of allergic diseases: A randomized, double-blind, placebo-controlled trial. J Allergy Clin Immunol 2007, 119:192-198.

121. Rochat F, Cherbut C, Barclay D, Puccio G, Fazzolari-Nesci A, Grathwohl D, Haschke F: A whey-predominant formula induces fecal microbiota similar to that found in breast-fed infants. Nutr Res 2007, 27:735-740.

122. Chou I, Kuo H, Chang J, Wu S, Chiu H, Su B, Lin H: Lack of effects of oral probiotics on growth and neurodevelopmental outcomes in preterm very low birth weight infants. J Pediatr 2010, 156:393-396.

123. Hol J, van Leer EHG, Elink Schuurman BEE, de Ruiter LF, Samsom JN, Hop W, Neijens HJ, de Jongste JC, Nieuwenhuis EES: The acquisition of tolerance toward cow's milk through probiotic supplementation: A randomized, controlled trial. J Allergy Clin Immunol 2008, 121:1448-1454.

124. Chandra RK: Effect of lactobacillus on the incidence and severity of acute rotavirus diarrhoea in infants, A prospective placebo-controlled doubleblind study. Nutr Res 2002, 22:65-69.

125. Karvonen AV, Sinkiewicz G, Connolly E, Vesikari T: Safety and colonization of the probiotic Lactobacillus reuteri ATCC 55730 in newborn infants and premature infants. In Clinical study 1. Stockholm, Sweden: BioGaia AB Research Laboratories; 1999. Unpublished.

126. Kukkonen K, Savilahti E, Haahtela T, Juntunen-Backman K, Korpela R, Poussa $T$, Tuure $T$, Kuitunen M: Long-term safety and impact on infection rates of postnatal probiotic and prebiotic (synbiotic) treatment: Randomized, double-blind, placebo-controlled trial. Pediatrics 2008, 122:8-12.

127. Taipale T, Pienihkkinen K, Isolauri E, Larsen C, Brockmann E, Alanen P, Jokela J, Söderling E: Bifidobacterium animalis subsp. lactis BB-12 in reducing the risk of infections in infancy. Br J Nutr 2011, 105:409-416.

128. Euler AR, Mitchell DK, Kline R, Pickering LK: Prebiotic effect of fructooligosaccharide supplemented term infant formula at two concentrations compared with unsupplemented formula and human milk. J Pediatr Gastroenterol Nutr 2005, 40:157-164.

129. Isolauri E, Arvola T, Sutas Y, Moilanen E, Salminen S: Probiotics in the management of atopic eczema. Clin Exp Allergy 2000, 30:1604-1610.

130. Lin H-C, Su B-H, Chen A-C, Lin T-W, Tsai C-H, Yeh T-F, Oh W: Oral probiotics reduce the incidence and severity of necrotizing enterocolitis in very low birth weight infants. Pediatrics 2005, 115:1-4.

131. Karvonen AV, Sinkiewicz G, Connolly E, Vesikari T: Safety and colonization of the probiotic Lactobacillus reuteri ATCC 55730 in newborn infants and premature infants. In Clinical study 2. Stockholm, Sweden: BioGaia AB Research Laboratories; 2001. Unpublished.

132. Taylor SN, Basile LA, Ebeling M, Wagner CL: Intestinal permeability in preterm infants by feeding type: Mother's milk versus formula. Breast Med 2009, 4:11-15

133. Hoyos $A B$ : Reduced incidence of necrotizing enterocolitis associated with enteral administration of lactobacillus acidophilus and bifidobacterium infantis to neonates in an intensive care unit. Int J Infect Dis 1999, 3:197-202

134. Nopchinda S, Varavithya W, Phuapradit P, Sangchai R, Suthutvoravut U, Chantraruksa V, Haschke F: Effect of bifidobacterium Bb12 with or without streptococcus thermophilus supplemented formula on nutritional status. J Med Assoc Thai 2002, 85(Suppl 4):S1225-S1231.

135. Manzoni $\mathrm{P}$, Mostert H, Leonessa ML, Priolo C, Farina D, Monetti C, Latino MA, Gomirato G: Oral supplementation with lactobacillus casei subspecies rhamnosus prevents enteric colonization by candida species in preterm neonates: A randomized study. Clin Infect Dis 2006, 42:1735-1742.

136. Karvonen AV, Sinkiewicz G, Connolly E, Vesikari T: Safety and colonization of the probiotic Lactobacillus reuteri ATCC 55730 in new Born and premature infants. In Clinical study 3. Stockholm, Sweden: BioGaia AB Research Laboratories; 2002. Unpublished.

137. Thibault $H$, Aubert-Jacquin C, Goulet O: Effects of long-term consumption of a fermented infant formula (with bifidobacterium breve c50 and streptococcus thermophilus 065) on acute diarrhea in healthy infants. J Pediatr Gastroenterol Nutr 2004, 39:147-152.

138. Kim S, Da HL, Meyer D: Supplementation of infant formula with native inulin has a prebiotic effect in formula-fed babies. Asia Pac J Clin Nutr 2007, 16:172-177.

139. Rivero M, Chifre R, Roca A, Conde M, Rodriguez M, Sanamaria A: Effect of a new infant formulae enriched prebiotics, probiotics, nucleotides and LC-PUFA on infants recovery after an infection. [abstract PC28]. J Pediatr Gastroenterol Nutr 2004, 39(Suppl 1)):19522. 
140. Rinne M, Kalliomäki M, Salminen S, Isolauri E: Probiotic intervention in the first months of life: Short-term effects on gastrointestinal symptoms and long-term effects on gut microbiota. J Pediatr Gastroenterol Nutr 2006, 43:200-205.

141. Li Y, Shimizu T, Hosaka A, Kaneko N, Ohtsuka Y, Yamashiro Y: Effects of bifidobacterium breve supplementation on intestinal flora of low birth weight infants. Pediatr Int 2004, 46:509-515.

142. Lee SJ, Cho SJ, Park EA: Effects of probiotics on enteric flora and feeding tolerance in preterm infants. Neonatology 2007, 91:174-179.

143. Urao M, Fujimoto T, Lane GJ, Seo G, Miyano T: Does probiotics administration decrease serum endotoxin levels in infants? J Pediatr Surg 1999, 34:273-276.

144. Samanta M, Sarkar M, Ghosh P, Ghosh JK, Sinha MK, Chatterjee S: Prophylactic probiotics for prevention of necrotizing enterocolitis in very low birth weight newborns. J Trop Pediatr 2009, 55:128-131.

145. Panigrahi P, Parida S, Pradhan L, Mohapatra SS, Misra PR, Johnson JA, Chaudhry R, Taylor S, Hansen NI, Gewolb IH: Long-term colonization of a lactobacillus plantarum synbiotic preparation in the neonatal gut. J Pediatr Gastroenterol Nutr 2008, 47:45-53.

146. Lidestri M, Agosti M, Marini A, Boehm G: Oligosaccharides might stimulate calcium absorption in formula-fed preterm infants. Acta Paediatr 2003, 91 (441):91-92.

147. Van Der Aa LB, Heymans HS, Van Aalderen WM, Sillevis Smitt JH, Knol J, Ben Amor K, Goossens DA, Sprikkelman AB: Effect of a new synbiotic mixture on atopic dermatitis in infants: A randomized-controlled trial. Clin Exp Allergy 2010, 40:795-804.

148. Rojas MA, Lozano JM, Rojas MX, Rodriguez VA, Rondon MA, Bastidas JA, Perez LA, Rojas C, Ovalle O, Garcia-Harker JE, Tamayo ME, Ruiz GC, Ballesteros A, Archila MM, Arevalo M: Prophylactic probiotics to prevent death and nosocomial infection in preterm infants. Pediatrics 2012 130:e1113-e1120.

149. Marini A, Negretti F, Boehm G, Li Destri M, Clerici-Bagozzi D, Mosca F, Agost M: Pro- and pre-biotics administration in preterm infants: Colonization and influence on faecal flora. Acta Paediatr 2003, 91(441):80-81.

150. Waligora-Dupriet A-J, Campeotto F, Nicolis I, Bonet A, Soulaines P, Dupont C, M- B: Effect of oligofructose supplementation on gut microflora and well-being in young children attending a day care centre. Int J Food Microbiol 2007, 113:108-113.

151. Taylor AL, Dunstan JA, Prescott SL: Probiotic supplementation for the first 6 months of life fails to reduce the risk of atopic dermatitis and increases the risk of allergen sensitization in high-risk children: $A$ randomized controlled trial. J Allergy Clin Immunol 2007, 119:184-191.

152. Rigo J, Pieltain C, Studzinski F: Growth, weight gain composition and mineral accretion in term infants fed a new experimental formula containing hydrolysed protein, $\beta$-palmitate and prebiotics. Pediatrika 2001, 21:39-48.

153. Wang C, Shoji H, Sato H, Nagata S, Ohtsuka Y, Shimizu T, Yamashiro Y: Effects of oral administration of bifidobacterium breve on fecal lactic acid and short-chain fatty acids in low birth weight infants. $J$ Pediatr Gastroenterol Nutr 2007, 44:252-257.

154. Underwood MA, Salzman NH, Bennett SH, Barman M, Mills DA, Marcobal A Tancredi DJ, Bevins CL, Sherman MP: A randomized placebo-controlled comparison of 2 prebiotic/probiotic combinations in preterm infants: Impact on weight gain, intestinal microbiota, and fecal short-chain fatty acids. J Pediatr Gastroenterol Nutr 2009, 48:216-225.

155. Savino F, Cresi F, Maccario S, Cavallo F, Dalmasso P, Fanaro S, Oggero R, Vigi $\checkmark$, Silvestro L: "Minor" feeding problems during the first months of life: Effect of a partially hydrolysed milk formula containing fructo- and galacto-oligosaccharides. Acta Paediatr 2003, 91:86-90

156. Sepp E, Mikelsaar M, Salminen S: Effect of administration of lactobacillus casei strain GG on the gastrointestinal microbiota of newborns. Microb Ecol Health Dis 1993, 6:309-314.

157. Alfaleh KM, Bassler D: Probiotics for prevention of necrotizing enterocolitis in preterm infants. In Cochrane Database of Systematic Reviews 2008, Issuel. Art. No:CD005496.DOI:10.1002/14651858.CD005496.pub2.

158. Mugambi MN, Musekiwa A, Lombard M, Young T, Blaauw R: Synbiotics, probiotics or prebiotics in infant formula for full term infants: $A$ systematic review. Nutr J 2012, 11(1):Article number 8. DOI:10.1186/14752891-11-81.
159. Mugambi MN, Musekiwa A, Lombard M, Young T, Blaauw R: Probiotics, prebiotics infant formula use in preterm or low birth weight infants: A systematic review. Nutr J 2012, 11(1):Article number 58. DOl:10.1186/1475-2891-11-58.

160. Lundh A, Sismondo S, Lexchin J, Busuioc OA, Bero L: Industry sponsorship and research outcome. Cochrane Database Syst Rev 2012, 12: . DOl: 10.1002/14651858.MR000033.pub2.

161. Bhandari M, Busse JW, Jackowski D, Montori VM, Schünemann $H$, Sprague S, Mears D, Schemitsch EH, Heels-Ansdell D, Devereaux PJ: Association between industry funding and statistically significant pro-industry findings in medical and surgical randomized trials. Can Med Assoc J 2004, 170:477-480.

162. Lexchin J, Bero LA, Djulbegovic B, Clark O: Pharmaceutical industry sponsorship and research outcome and quality: Systematic review. $\mathrm{Br}$ Med J 2003, 326:1167-1170.

163. Nestle M: Food company sponsorship of nutrition research and professional activities: A conflict of interest? Public Health Nutr 2001, 4:1015-1022.

doi:10.1186/1471-2288-13-137

Cite this article as: Mugambi et al:: Association between funding source, methodological quality and research outcomes in randomized controlled trials of synbiotics, probiotics and prebiotics added to infant formula: A Systematic Review. BMC Medical Research Methodology 2013 13:137.

\section{Submit your next manuscript to BioMed Central and take full advantage of:}

- Convenient online submission

- Thorough peer review

- No space constraints or color figure charges

- Immediate publication on acceptance

- Inclusion in PubMed, CAS, Scopus and Google Scholar

- Research which is freely available for redistribution

Submit your manuscript at www.biomedcentral.com/submit
C Biomed Central 